\title{
BASS SERIES OF LOCAL RING HOMOMORPHISMS OF FINITE FLAT DIMENSION
}

\author{
LUCHEZAR L. AVRAMOV, HANS-BJØRN FOXBY, AND JACK LESCOT \\ Dedicated to Robert Fossum on his fiftieth birthday
}

\begin{abstract}
Nontrivial relations between Bass numbers of local commutative rings are established in case there exists a local homomorphism $\phi: R \rightarrow S$ which makes $S$ into an $R$-module of finite flat dimension. In particular, it is shown that an inequality $\mu_{R}^{i+\operatorname{depth} R} \leq \mu_{s}^{i+\operatorname{depth} S}$ holds for all $i \in \mathbb{Z}$. This is a consequence of an equality involving the Bass series $I_{R}^{M}(t)=\sum_{i \in \mathbf{Z}} \mu_{R}^{i}(M) t^{i}$ of a complex $M$ of $R$-modules which has bounded above and finite type homology and the Bass series of the complex of $S$-modules $M \stackrel{\otimes}{=}_{R} S$, where $\otimes$ denotes the derived tensor product. It is proved that there is an equality of formal Laurent series $I_{s}^{M} \stackrel{\otimes}{=} S$ considered as a homomorphism of commutative differential graded rings. Coefficientwise inequalities are deduced for $I_{S} \stackrel{M \otimes}{=} S$, $(t)$, and Golod homomorphisms are characterized by one of them becoming an equality.
\end{abstract}

\section{INTRODUCTION}

This paper describes relations between the Bass series $I_{R}(t)$ and $I_{S}(t)$, when there is a local homomorphism $\phi: R \rightarrow S$ of local noetherian rings making $S$ into an $R$-module of the finite flat dimension. Recall, that when $R$ has maximal ideal $\mathfrak{m}$ and residue field $k=R / \mathfrak{m}$, and $M$ is a finitely generated $R$ module, the Bass series of $M$ is the formal power series $I_{R}^{M}(t)=\sum_{i \geq 0} \mu_{R}^{i}(M) t^{i}$ where $\mu_{R}^{i}(M)$ is the dimension of the $h$-vector space $\operatorname{Ext}_{R}^{i}(\not k, M)$ (and $I_{R}(t)$ stands for $\left.I_{R}^{R}(t)\right)$. This series is of interest since the Bass numbers $\mu_{R}^{i}(=$ $\mu_{R}^{i}(R)$ ) contain important information on the ring $R$. The bridge between $I_{R}(t)$ and $I_{S}(t)$ is provided by the invariants of the $D G$ fiber $F=F(\phi)$ of the homomorphism $\phi$. This fiber was introduced by Avramov in [1] as a differential graded algebra whose homology is naturally isomorphic to $\operatorname{Tor}^{R}(\mathcal{E}, S)$ (cf. $\S 3$ below). In particular, $F$ is augmented to the residue field $\ell=S / \mathfrak{n}$ of $S$. This allows the construction of the $\ell$-vector space $\operatorname{Ext}_{F}^{i}(\ell, F)$ (cf. [7], [5], and $\S 1$ below). Let the $i$ th Bass number $\mu_{F}^{i}$ of $F$ be the dimension of this $\ell$-vector

Received by the editors November 20, 1989 and, in revised form, August 9, 1990.

1980 Mathematics Subject Classification (1985 Revision). Primary 13D03; Secondary 13D25, $18 \mathrm{G} 10,18 \mathrm{G} 15,55 \mathrm{~T} 20$.

Research of the first author was supported, in part, by Contract $884 / 88$ with the Ministry of Culture, Science, and Education of Bulgaria.

Research of the second author was supported, in part, by SNF, the Danish Research Council. 
space, and set $\mu_{\phi}^{i}=\mu_{F}^{i}$ and $I_{\phi}(t)=\sum_{i \in \mathbb{Z}} \mu_{\phi}^{i} t^{i}:$ this is a formal Laurent series in case $\mu_{\phi}^{i}$ is finite for all $i \in \mathbb{Z}$ and $\mu_{\phi}^{i}=0$ for $i$ small enough.

Theorem A. Let $\phi: R \rightarrow S$ be a local ring homomorphism of finite flat dimension and with $D G$ fiber $F(\phi)$. All the Bass numbers $\mu_{\phi}^{i}$ are then finite and vanish for $i$ small enough, and there is an identity of formal Laurent series

$$
I_{S}(t)=I_{R}(t) I_{\phi}(t) \text {. }
$$

In fact, we prove in $\S 5$ a more general result involving Bass series of modules. When $\phi$ is flat $F(\phi)$ can be replaced by the local ring $S / \mathrm{m} S$. This shows that the result of $\S 5$ extends the one in [12] from flat homomorphisms to homomorphisms of finite flat dimension.

The formula for the Bass series gives nontrivial lower and upper bounds for the Bass series (numbers) of $S$ in terms of those of $R$.

\section{Theorem B.}

(1) With $\phi$ as above the inequalities

$$
\mu_{R}^{i+\text { depth } R} \leq \mu_{S}^{i+\text { depth } S},
$$

hold for all $i \in \mathbb{Z}$.

(2) Assume furthermore, that $S / \mathrm{m} S$ is artinian. Unless $\phi$ is flat and $S / \mathrm{m} S$ is a field, the following coefficient wise inequality holds:

$$
I_{S}(t) \ll I_{R}(t) \frac{\sum_{i=0}^{f}\left(\text { length }_{S} \operatorname{Tor}_{i}^{R}(\not k, S)\right) t^{-i}-t-1}{1+t-\sum_{i=0}^{f}\left(\text { length }_{S} \operatorname{Tor}_{i}^{R}(\not k, S)\right) t^{i+1}}
$$

where $f=\operatorname{fd}_{R} S$, the flat dimension of the $R$-module $S$.

When $\phi$ is flat and $S / \mathrm{m} S$ is a field, it is obvious that equality holds in (*) for all $i$. Those $\phi$ which have equality in $(*)$ for all $i \in \mathbb{Z}$ form a large and interesting class, which is studied in detail in [4].

Next we discuss when equality holds in $(* *)$. To this end, recall that for an arbitrary local ring homomorphism $\phi$ with $S / \mathrm{m} S$ artinian there is an inequality of Poincaré series:

$$
P^{S}(t) \ll P^{R}(t) \frac{1}{1+t-\sum_{i=0}^{f}\left(\operatorname{length}_{S} \operatorname{Tor}_{i}^{R}(\not k, S)\right) t^{i+1}},
$$

(where $\left.P^{R}(t)=\sum_{i \geq 0}\left(\operatorname{dim}_{\ell} \operatorname{Tor}_{i}^{R}(\not k, \ell)\right) t^{i}\right)$. In case equality holds, $\phi$ is called a standard Golod homomorphism. (In general, a Golod homomorphism is defined by the condition that $H(F(\phi))$ be an algebra with trivial Massey products. If length Tor $^{R}(\mathcal{k}, S) \neq 2$ then $\phi$ is Golod if and only if $\phi$ is standard Golod, cf. [2].)

Theorem C. Let $\phi: R \rightarrow S$ be a local ring homomorphism of finite flat dimension $f$ with $S / \mathrm{m} S$ artinian. Assume furthermore that $\phi$ is not flat with $S / \mathrm{m} S$ a field. There is then equality in (**) if and only if $\phi$ is Golod.

In fact, the results of $\S 5$ are generalizations of Theorems $\mathrm{A}, \mathrm{B}$, and $\mathrm{C}$ in two directions: they deal with Bass series of complexes of $R$-modules and they avoid the artinian assumption on $S / \mathrm{m} S$.

Next we describe applications to a different setup. According to the Looking Glass Principle in [7] properties of the (co-)homology of local rings usually have 
some counterpart in rational homotopy theory. The results of this paper are no exception. Indeed, let $G$ be a topological space with finite Betti numbers $b_{i}=\operatorname{dim} H^{i}(G, \mathbb{Q})$, such that $b_{1}=0, b_{f} \neq 0$, and $b_{i}=0$ for $i>f$. If $G \rightarrow E \rightarrow B$ is a fibration such that $B$ is a simply connected space with $H^{*}(B, \mathbb{Q})$ of finite type, then the precise analog of Theorem $\mathrm{A}$ is provided by the recent result of Félix, Halperin, and Thomas [10]: There is an isomorphism

$$
\begin{aligned}
& \operatorname{Ext}_{C^{*}(E, \mathbb{Q})}\left(\mathbb{Q}, C^{*}(E, \mathbb{Q})\right) \\
& \quad \cong \operatorname{Ext}_{C^{*}(B, \mathbb{Q})}\left(\mathbb{Q}, C^{*}(B, \mathbb{Q})\right) \otimes_{\mathbb{Q}} \operatorname{Ext}_{C^{*}(G, \mathbb{Q})}\left(\mathbb{Q}, C^{*}(G, \mathbb{Q})\right),
\end{aligned}
$$

where $C^{*}(-, \mathbb{Q})$ denotes the singular cochain algebra with rational coefficients. The analogs of Theorems B and C we state in the absolute case only.

Theorem D. For $G$ as above, the following inequalities hold for the Laurent series $I_{G}(t)=\sum_{i \in \mathbb{Z}}\left(\operatorname{dim}_{\mathbb{Q}} \operatorname{Ext}_{C^{*}(G, \mathbb{Q})}^{-i}\left(\mathbb{Q}, C^{*}(G, \mathbb{Q})\right)\right) t^{i}$ :

$$
b_{f} t^{-f} \ll I_{G}(t) \ll \frac{\sum_{i=2}^{f} b_{i} t^{-i}-t^{-1}}{1-\sum_{i=2}^{f} b_{i} t^{i-1}} .
$$

Equality holds on the right-hand side if and only if $G$ is rationally equivalent to a wedge of spheres, namely $G_{0} \sim \bigvee_{i=2}^{f}\left(\bigvee^{b_{i}} S^{i}\right)$.

(There is a version of this result which holds for homology with coefficients in an arbitrary field, cf. (2.13). In [10] it is discussed when equality holds on the left-hand side.)

Out of the five section of this paper, the first and the third are introductory, and the second and the fourth are computations in differential graded homological algebra, while the applications to commutative algebra are given in $\S 5$. The main results of this paper have been announced in the note [6].

The statements of many of our results involve only classical invariants of commutative rings and modules, but their proofs depend in an essential way on the techniques of DG homological algebra. This supports our point of view that much is to be gained by embedding commutative algebra into differential graded commutative algebra. The sequel to this paper, [4], represents an even more transparent application of this principle.

\section{Summary of DG homological algebra}

Following earlier work of Eilenberg-Mac Lane and Cartan on the (co)homology of $K(\pi, n)$ 's, Eilenberg and Moore invented differential graded homological algebra, cf. Moore [18]. In particular, they constructed groups $\operatorname{Tor}_{i}^{R}(L, M)$ for bounded DG modules over a DG ring $R$ concentrated in nonnegative degrees. However, their technique does not provide the groups $\operatorname{Ext}_{R}^{i}(M, N)$ unless connectivity restrictions are present. This is not the case for the DG rings and DG modules of this paper, so we use the construction of the derived functors introduced in [7], which works in general and is based on the notion of semifree DG modules. Below we present an outline of this approach using the familiar idiom of projective resolutions. This presentation is rather expansive because a comprehensive account is only in preparation, and hence it will only be available in the literature later (in [5]).

A $G$ ring is a collection of abelian groups $R=\left(R_{i}\right)_{i \in \mathbb{Z}}$ together with pairings: $R_{i} \times R_{j} \rightarrow R_{i+j}$ (for $i, j \in \mathbb{Z}$ ) subject to the usual requirements of associativity, 
distributivity, and existence of a unit $i \in R_{0}$. In particular, $R_{0}$ is a ring, and $R_{i}$ is an $R_{0}$-module for all $i \in \mathbb{Z}$. An element of $R$ is an element of $R_{i}$ for some $i$. In this paper we do not consider the direct sum $\coprod_{i \in \mathbb{Z}} R_{i}$ (with one exception in a cautionary remiark). This is the reason for our usage of the abbreviation $G$ ring instead of the expression graded ring.

A homomorphism of $\mathrm{G}$ rings $\phi: R \rightarrow S$ is a collection of additive maps $\left(\phi_{i}: R_{i} \rightarrow S_{i}\right)_{i \in \mathbb{Z}}$ such that $\phi_{0}(1)=1$, and $\phi_{m+n}(x y)=\phi_{m}(x) \phi_{n}(y)$ for $x \in R_{m}$ and $y \in R_{n}$. In particular, $\phi_{0}$ is a homomorphism of rings, and $\phi_{i}$ is a homomorphism of $R_{0}$-modules for all $i \in \mathbb{Z}$.

A left $G R$-module is a collection of abelian groups $M=\left(M_{j}\right)_{j \in \mathbb{Z}}$ together with pairings: $R_{i} \times M_{j} \rightarrow M_{i+j}$ (for $i, j \in \mathbb{Z}$ ) subject to the usual conditions. In particular, $M_{i}$ is an $R_{0}$-module for all $i \in \mathbb{Z}$. An element of $M$ is an element of $M_{i}$ for some $i$. A right $G R$-module is defined by interchanging the factors. $G$ modules are left $G$ modules unless otherwise specified.

In this setup (which is that of Moore [18] and Mac Lane [17]) each nonzero element of $R$ or $M$ has a unique degree denoted by || . That is, $|x|=i$, if $x \neq 0$ and $x$ belongs to $R_{i}$ or to $M_{i}$. A G $R$-module $M$ is said to be bounded above (respectively: bounded below, bounded), if there exits an $n \in \mathbb{Z}$ such that $M_{i}=0$ for $i>n$ (respectively: for $i<n$, for $|i|>n$ ).

A $G R$-module $M$ is said to be of $G$ finite type if there exists a set of generators $E$ for $M$ such that $E_{i}=\{e \in E|| e \mid=i\}$ is finite for each $i \in \mathbb{Z}$. If there exists an $n \in \mathbb{Z}$ such that $E_{i}=\varnothing$ for $i>n$ (respectively: for $i<n$, $|i|>n$ ) then $M$ is said to be of bounded above (respectively: bounded below, bounded) type.

A homomorphism of degree $n$ of left $\mathrm{G} R$-modules $\alpha: M \rightarrow N$ is a collection of additive maps $\left(\alpha_{i}: M_{i} \rightarrow N_{i+n}\right)_{i \in \mathbb{Z}}$ satisfying the identities

$$
\alpha(r m)=(-1)^{|r| n} r \alpha(m) \text { for } r \in R \text { and } m \in M \text {. }
$$

Remark. Here is the first instance of the general rule which we (have tried to) follow throughout the paper: When, in an expression, two elements $a$ and $b$ are transposed the sign $(-1)^{|a||b|}$ appears. (In the example above $|\alpha|=n$.)

We denote by $\operatorname{Hom}_{R}(M, N)_{n}$ the abelian group of homomorphisms: $M \rightarrow$ $N$ of degree $n$, and $\operatorname{Hom}_{R}(M, N)$ denotes the $\mathrm{G} \mathbb{Z}$-module of homomorphisms $\left(\operatorname{Hom}_{R}(M, N)_{n}\right)_{n \in \mathbb{Z}} \cdot\left(\right.$ Caution: $\bigsqcup_{i \in \mathbb{Z}} \operatorname{Hom}_{R}(M, N)_{i}$ is not (in general) the group of $\left(\coprod_{i \in \mathbb{Z}} R_{i}\right)$-homomorphisms: $\bigsqcup_{i \in \mathbb{Z}} M_{i} \rightarrow \bigsqcup_{i \in \mathbb{Z}} N_{i}$.)

A morphism $\alpha: M \rightarrow N$ of $\mathrm{G} R$-modules is a homomorphism of degree zero. A left $G R$-module $N$ is said to be $G$-projective (respectively, $G$-injective) if the functor $\operatorname{Hom}_{R}(N,-)$ (respectively, $\operatorname{Hom}_{R}(-, N)$ ) preserves exact sequences of morphisms of left $\mathrm{G} R$-modules. A left $\mathrm{G} R$-module $F$ is $G$-flat if $-\otimes_{R} F$ preserves exact sequences of right $\mathrm{G} R$-modules. A G-projective $\mathrm{G}$ module is G-flat.

Each $G R$-module $M$ has a $G$-projective resolution $\mathbb{P}$, that is, a complex of morphisms of projective $G$ modules

$$
\mathbb{P}=\cdots \rightarrow P_{(i)} \stackrel{\partial}{\rightarrow} P_{(i-1)} \rightarrow \cdots \rightarrow P_{(1)} \stackrel{\partial}{\rightarrow} P_{(0)} \rightarrow 0 \rightarrow \cdots,
$$

with $H_{(0)}(\mathbb{P})$ isomorphic to $M$ and $H_{(i)}(\mathbb{P})=0$ for $i \neq 0$. The $\mathrm{G} \mathbb{Z}$ module $\operatorname{Ext}_{R}^{i}(M, N)$ is defined up to unique isomorphism by $\operatorname{Ext}_{R}^{i}(M, N)_{j}$ $=H_{(-i)}\left(\operatorname{Hom}_{R}(\mathbb{P}, N)_{j}\right)$, when $\mathbb{P}$ is a G-projective resolution of $M$, and 
$\operatorname{Hom}_{R}(\mathbb{P}, N)$ is the complex of $\mathrm{G} R$-modules

$$
\cdots \rightarrow 0 \rightarrow \operatorname{Hom}_{R}\left(P_{(0)}, N\right) \stackrel{\operatorname{Hom}_{R}(\partial, N)}{\longrightarrow} \operatorname{Hom}_{R}\left(P_{(1)}, N\right) \rightarrow \cdots .
$$

For $\mathrm{G} \mathbb{Z}$-modules $L$ and $M$ the tensor product $L \otimes_{\mathbb{Z}} M$ is the $\mathrm{G} \mathbb{Z}$-module defined by $\left(L \otimes_{\mathbb{Z}} M\right)_{i}=\bigsqcup_{p+q=i} L_{p} \otimes_{\mathbb{Z}} M_{q}$. For a right $\mathrm{G} R$-module $L$ and a left $\mathrm{G} R$-module $M$ the tensor product $L \otimes_{R} M$ is the cokernel of the natural morphism of $G \mathbb{Z}$-modules

$$
L \otimes_{\mathbb{Z}} R \otimes_{\mathbb{Z}} M \rightarrow L \otimes_{\mathbb{Z}} M \quad(l \otimes r \otimes m \mapsto l r \otimes m-l \otimes r m) .
$$

As usual $\operatorname{Tor}_{1}^{R}(L, M)$ is the $G \mathbb{Z}$-module defined up to unique isomorphism by $\operatorname{Tor}_{i}^{R}(L, M)_{j}=H_{(i)}\left(\left(L \otimes_{R} \mathbb{P}\right)_{j}\right)$ when $\mathbb{P}$ is a G-projective resolution of $M$.

A (left) derivation of degree $n$ of the $G$ ring $R$ is a collection of additive maps $\partial=\left(\partial_{i}: R_{i} \rightarrow R_{i+n}\right)_{i \in \mathbb{Z}}$ satisfying the Leibniz rule

$$
\partial(r s)=\partial(r) s+(-1)^{|r| n} r \partial s .
$$

A G ring $R$ together with a degree -1 derivation $\partial$ of square zero is called a $D G$ ring. The derivation $\partial$ is then referred to as the differential of $R$. The $G$ ring underlying the DG ring $R$ is denoted by $R^{\#}$.

A homomorphism of $D G$ rings $\phi: R \rightarrow S$ is a homomorphism of $\mathrm{G}$ rings satisfying $\phi \partial=\partial \phi$. A homomorphism of DG rings $\phi: R \rightarrow S$ such that $H(\phi): H(R) \rightarrow H(S)$ is an isomorphism is said to be a homology isomorphism.

If $R$ is a DG ring, a left (respectively: right) DG $R$-module $M$ is a left (respectively: right) $\mathrm{G} R^{\#}$-module $M^{\#}$ together with a degree -1 additive endomorphism $\partial: M \rightarrow M$, such that $\partial^{2}=0$ and

$$
\left.\partial(r x)=\partial(r) x+(-1)^{|r|} r \partial x \quad \text { (respectively: } \partial(x r)=\partial(x) r+(-1)^{|x|} x \partial r\right),
$$

for all $r \in R$ and $x \in M ; \partial$ is called the differential of $M$.

Remark. Throughout this paper notions with DG are natural extensions of the usual notions from module theory to a differential graded (DG) setup. However, the inverse passage from the DG framework to that of module theory is not always reflected in a deletion of DG in the notation.

For example, a ring $A$ may (and will) be considered as a DG ring $A$ by setting $A_{0}=A$ and $A_{i}=0$ for $i \neq 0$. We shall refer to this by saying, that $A$ is concentrated in degree zero. Similarly a left $A$-module can be considered as a left DG $A$-module. However, a left DG $A$-module is a complex of left $A$-modules! In this situation we use the two expressions, DG $A$-module and complex of A-modules, interchangeably.

A morphism $\alpha: M \rightarrow N$ of DG $R$-modules is a morphism: $M^{\#} \rightarrow N^{\#}$ of G $R^{\#}$-modules such that $\alpha \partial=\partial \alpha$.

The cycles of $M$, the boundaries of $M$, and the homology of $M$ are defined, as usual, by

$$
Z(M)=\{a \in M \mid \partial a=0\} ; \quad B(M)=\{\partial b \mid b \in M\} ; \quad H(M)=Z(M) / B(M),
$$

respectively.

The homology $H(R)$ is a $\mathrm{G}$ ring and $H$ is a functor from DG $R$-modules to $\mathrm{G} H(R)$-modules. The homology class of a cycle $z$ is denoted [ $z]$. A DG $R$-module $M$ is said to be homologically trivial, if $H(M)=0$. A morphism $\alpha: M \rightarrow N$ is said to be a homology isomorphism, if $H(\alpha): H(M) \rightarrow H(N)$ is 
an isomorphism (of $\mathrm{G} H(R)$-modules). A homology isomorphism is indicated by the symbol $\simeq$ next to its arrow, while $\cong$ is our symbol for isomorphisms.

Two morphisms $\alpha, \beta: M \rightarrow N$ are said to be $R$-homotopic, if there exists a $\gamma \in \operatorname{Hom}_{R}(M, N)_{1}$ such that $\partial \gamma+\gamma \partial=\alpha-\beta$; in this case we write $\alpha \sim \beta$. Note that this implies $H(\alpha)=H(\beta)$.

For DG $R$-modules $M$ and $N$ a DG $\mathbb{Z}$-module $\operatorname{Hom}_{R}(M, N)$ is defined by

$$
\begin{gathered}
\operatorname{Hom}_{R}(M, N)^{\#}=\operatorname{Hom}_{R^{\#}}\left(M^{\#}, N^{\#}\right) \text { and } \\
\partial(\alpha)(m)=\partial(\alpha m)-(-1)^{|\alpha|} \alpha(\partial m) \text { for } \alpha \in \operatorname{Hom}_{R}(M, N) \text { and } m \in M .
\end{gathered}
$$

For a right DG $R$-module $L$ and a left DG $R$-module $M$ a DG $\mathbb{Z}$-module $L \otimes_{R} M$ is defined by

$$
\begin{gathered}
\left(L \otimes_{R} M\right)^{\#}=L^{\#} \otimes_{R^{\#}} M^{\#} \text { and } \\
\partial(l \otimes m)=\partial(l) \otimes m+(-1)^{|l|} l \otimes \partial m \quad \text { for } l \in L \text { and } m \in M .
\end{gathered}
$$

A DG module $P$ such that $\operatorname{Hom}_{R}(P,-)$ preserves homology isomorphisms,

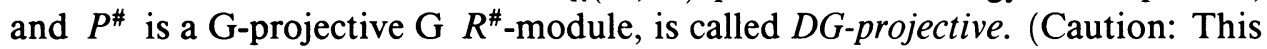
notion is less restrictive than the one in [18].)

A DG module $I$ such that $\operatorname{Hom}_{R}(-, I)$ preserves homology isomorphisms, and $I^{\#}$ is a G-injective $\mathrm{G} R^{\#}$-module, is called $D G$-injective.

A DG module $F$ such that $-\otimes_{R} F$ preserves homology isomorphisms, and $F^{\#}$ is a G-flat $G R^{\#}$-module, is called $D G$-flat. (Caution: This notion is more restrictive than the one in [10, Appendix].)

Remark. Assume that $R=A$ is concentrated in degree zero. A bounded below DG $A$-module $P$ is a DG-projective (respectively, DG-flat) DG module if and only if each $P_{i}$ is a projective (respectively, flat) $A$-module. A bounded above DG $A$-module $I$ is a DG-injective DG module if and only if each $I_{i}$ is an injective $A$-module.

A homology isomorphism of DG $R$-modules: $P \cong M$ with $P$ DG-projective (respectively: DG-flat) is said to be a $D G$-projective (respectively: $D G$-flat) resolution of $M$ (over $R$ ). A homology isomorphism of DG $R$-modules: $M \cong I$ with $I$ DG-injective is said to be a $D G$-injective resolution of $M$ (over $R$ ).

Remark. Assume that $R=A$ is concentrated in degree zero, and $M$ is an $R$-module. The usual resolutions of homological algebra are then resolutions in the sense above.

(1.1) Every $D G R$-module $M$ has a $D G$-projective resolution $\varepsilon: P \cong M$ with $\varepsilon$ surjective. Every $D G R$-module $M$ has a $D G$-injective resolution $\eta: M \stackrel{\cong}{\rightrightarrows}$ with $\eta$ injective.

(1.2) Let $K$ be a $D G$-projective (respectively, $D G$-injective) $D G R$-module. Then to each morphism $\alpha: K \rightarrow N$ (respectively, $\gamma: M \rightarrow K$ ) and each homology isomorphism $\beta: M \stackrel{\cong}{\rightrightarrows} N$ there exists a morphism $\gamma: K \rightarrow M$ (respectively, $\alpha: N \rightarrow K$ ), such that $\alpha \sim \beta \gamma$ (respectively, $\alpha \beta \sim \gamma$ ), that is, such that the diagram

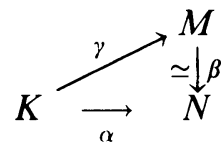

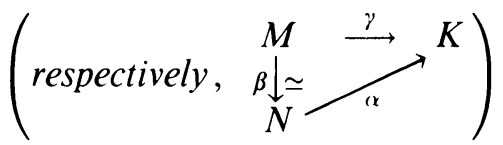


commutes up to homotopy. Furthermore this $\gamma$ (respectively, $\alpha$ ) is necessarily unique up to $R$-homotopy, that is, if $\gamma^{\prime}$ (respectively, $\alpha^{\prime}$ ) satisfies $\alpha \sim \beta \gamma^{\prime}$ (respectively, $\alpha^{\prime} \beta \sim \gamma$ ), then $\gamma^{\prime} \sim \gamma$ (respectively, $\alpha^{\prime} \sim \alpha$ ).

(1.3) Each DG-projective DG R-module is DG-flat.

(1.4) If $\gamma: P^{\prime} \cong P$ is a homology isomorphism of left DG-projective (respectively: DG-flat) $D G R$-modules, then for any left $D G R$-module $N$ (respectively: right $D G R$-module $L$ ), $\operatorname{Hom}(\gamma, N)$ (respectively: $L \otimes \gamma$ ) is a homology isomorphism.

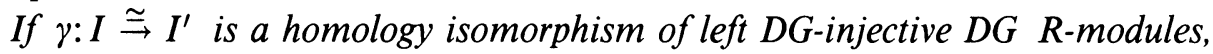
then $\operatorname{Hom}(\gamma, N)$ is a homology isomorphism for any left $D G R$-module $M$.

We let $\underline{\underline{H}}_{R}(M, N)$ (respectively: $L \underline{\underline{\otimes}}_{R} M$ ) denote any of the DG $\mathbb{Z}$ modules $\operatorname{Hom}_{R}(P, I)$ (respectively: $Q \otimes_{R} P$ ), where $P$ is a DG-projective resolution of the DG $R$-module $M, I$ is a DG-injective resolution of the DG $R$-module $N$, and $Q$ is a DG-projective resolution of the right DG $R$ module $L$. By (1.2) any two such DG modules are connected by homology isomorphisms, which themselves are defined uniquely up to homotopy. Thus $H\left(\underline{\underline{H}}_{R}(M, N)\right)$ (respectively: $\left.H\left(L \underline{\underline{\otimes}}_{R} M\right)\right)$ is defined up to unique isomorphism.

\section{Definition.}

and

$$
\operatorname{Ext}_{R}^{i}(M, N)=H_{-i}\left(\underline{\underline{H}}_{R}(M, N)\right)
$$

$$
\operatorname{Tor}_{i}^{R}(L, M)=H_{i}\left(\underline{\underline{\otimes}}_{R} M\right) .
$$

Remark. Assume that $R=A$ is concentrated in degree zero. If $M$ and $N$ are $R$-modules, these constructions give the standard notions. If $M$ and $N$ are complexes of $R$-modules bounded on the appropriate side, then the constructions specialize to hyper(co)homology, cf. e.g. [14] or [11].

Let $L$ be a right DG $R$-module, and let $M$ and $N$ be left DG $R$-modules. Let also $R^{\prime}$ be a DG ring. Let $L^{\prime}$ be a right DG $R^{\prime}$-module, and let $M^{\prime}$ and $N^{\prime}$ be left DG $R^{\prime}$-modules. In order to describe the functoriality of Ext and Tor consider the following situation:

$\phi: R \rightarrow R^{\prime}$ is a homomorphism of DG rings,

$\alpha: L \rightarrow L^{\prime}$ is an additive morphism, such that $\alpha(l r)=\alpha(l) \phi(r)$ for $r \in R$ and $l \in L$,

$\beta: M \rightarrow M^{\prime}$ is an additive morphism, such that $\beta(r m)=\phi(r) \beta(m)$ for $r \in R$ and $m \in M$, and

$\gamma: N^{\prime} \rightarrow N$ is an additive morphism, such that $\gamma\left(\phi(r) n^{\prime}\right)=r \gamma\left(n^{\prime}\right)$ for $r \in R$ and $n^{\prime} \in N^{\prime}$.

Using (1.2) one constructs, as usual, homomorphisms of $\mathrm{G} \mathbb{Z}$-modules:

$$
\operatorname{Tor}^{\phi}(\alpha, \beta): \operatorname{Tor}^{R}(L, M) \rightarrow \operatorname{Tor}^{R^{\prime}}\left(L^{\prime}, M^{\prime}\right)
$$

and

$$
\operatorname{Ext}_{\phi}(\beta, \gamma): \operatorname{Ext}_{R^{\prime}}\left(M^{\prime}, N^{\prime}\right) \rightarrow \operatorname{Ext}_{R}(M, N) .
$$

Their main property, which we shall mostly use without specific reference in the rest of the paper, is given by the following theorem. 
(1.5) The $G \mathbb{Z}$-modules $\operatorname{Tor}^{R}(M, N)$ and $\operatorname{Ext}_{R}(M, N)$ are functorial in the triple $(M, R, N)$. When $\phi, \alpha, \beta$, and $\gamma$ are homology isomorphisms, then $\operatorname{Tor}^{\phi}(\alpha, \beta)$ and $\operatorname{Ext}_{\phi}(\beta, \gamma)$ are isomorphisms.

Furthermore, by the definitions and (1.3), we have

(1.6) The induced maps:

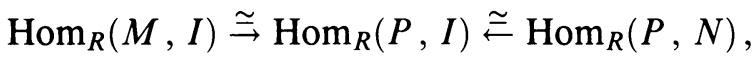

$$
\begin{aligned}
& Q \otimes_{R} M \cong Q \otimes_{R} P \cong \otimes_{R} P,
\end{aligned}
$$

are homology isomorphisms, which are defined uniquely up to homotopy.

After this short exposition of basic DG homological techniques, we pass to the discussion of more specific facts, which will be needed below.

(1.7) If $\phi: R \rightarrow S$ is a homomorphism of $D G$ rings making $S$ into a $D G$ projective (respectively: $D G$-flat) left $D G R$-module, and if $P$ is a DG-projective (respectively: $D G$-flat) left $D G S$-module, then $P$ is also DG-projective (respectively: DG-flat) as a left DG R-module.

An augmented $D G$ ring $\varepsilon_{R}: R \rightarrow \ell$ is a homomorphism of the DG ring $R$ into a field $\ell$ concentrated in degree zero, such that $k$ is the field of fractions of the image $\varepsilon_{R}(R)$. We shall often suppress $\varepsilon_{R}$ or $\ell$ (or both of them) from the notation. The field $b$ will always be considered as a left and right DG $R$-module with the structure given by $\varepsilon_{R}$. Of special interest are the $h$ vector spaces $\operatorname{Ext}_{R}^{i}(\not R, R)$. Let also $\varepsilon_{R^{\prime}}: R^{\prime} \rightarrow \ell^{\prime}$ be an augmented DG ring. A homomorphism of augmented DG rings: $R \rightarrow R^{\prime}$ is a homomorphism of the underlying DG rings such that $\left(\operatorname{Ker} \varepsilon_{R^{\prime}}\right) \cap R=\operatorname{Ker} \varepsilon_{R}$. When $R$ is a local ring (concentrated in degree zero) $\varepsilon_{R}$ will always denote the canonical surjection of $R$ onto the residue field.

(1.8) Let $\phi: R \rightarrow R^{\prime}$ be a homology isomorphism of augmented DG rings. The induced map: $k \rightarrow k^{\prime}$ is then an isomorphism, and the $k$-vector spaces $\operatorname{Ext}_{R}^{i}(\not{k}, R)$ and $\operatorname{Ext}_{R^{\prime}}^{i}\left(\ell^{\prime}, R^{\prime}\right)$ are canonically isomorphic.

Proof. The claim on the field extension is verified directly, and (1.5) provides isomorphisms

$$
\operatorname{Ext}_{R}^{i}(\not, R) \stackrel{\cong}{=} \operatorname{Ext}_{R}^{i}\left(\ell^{\prime}, R^{\prime}\right) \cong \operatorname{Ext}_{R^{\prime}}^{i}\left(\ell^{\prime}, R^{\prime}\right) .
$$

For a DG module $M$ over an augmented DG ring $R \rightarrow k$ the Bass number $\mu_{R}^{i}(M)$ is the (cardinal) number $\operatorname{dim}_{\ell} \operatorname{Ext}_{R}^{i}(\not{k}, M)\left(\operatorname{dim}_{\ell}\right.$ denotes dimension as a $\not$-vector space). The Bass "series" $I_{R}^{M}(t)$ is the "Laurent series"

$$
I_{R}^{M}(t)=\sum_{i \in \mathbb{Z}} \mu_{R}^{i}(M) t^{i}
$$

We set

$$
\operatorname{depth}_{R} M=\inf \left\{i \in \mathbb{Z} \mid \operatorname{Ext}_{R}^{i}(\not k, M) \neq 0\right\} \in\{-\infty\} \cup \mathbb{Z} \cup\{\infty\} .
$$

When $d=\operatorname{depth}_{R} M \in \mathbb{Z}$, we set

$$
\operatorname{DGtype}_{R} M=\operatorname{dim}_{\ell} \operatorname{Ext}_{R}^{d}(\not R, M) \in\{0\} \cup \mathbb{N} \cup\{\infty\} .
$$


We abbreviate $\operatorname{depth}_{R} R$ and DGtype ${ }_{R} R$ to depth $R$ and DGtype $R$, respectively.

Remark. If $M$ is a finitely generated module over a local noetherian ring $R$, then we recover the usual notion of depth; however for type we keep the DG in this case also, since type ${ }_{R} M$ is sometimes defined as $\operatorname{dim}_{\mathscr{k}} \operatorname{Ext}_{R}^{n}(\mathscr{k}, M)$ with $n=\operatorname{dim} M$.

More generally, for complexes of modules over a local ring, the following finiteness results hold, cf. [14] or [11].

(1.9) If $R$ is noetherian and concentrated in degree zero and $H(M)$ is bounded above, then $M$ has a bounded above DG-injective resolution. If, furthermore, all $H_{i}(M)$ are finitely generated, then $I_{R}^{M}(t)$ is a formal Laurent series, and $I_{R}^{M}(t) \neq$ 0 when $H(M) \neq 0$. In particular, $\operatorname{depth}_{R} M \in \mathbb{Z}$ and $\mathrm{DGtype}_{R} M<\infty$.

In some of our computations we shall have to carefully keep track of different module structures. To do this we use the standard language of bimodules. However, since the signs are important and may get complicated, we have collected the necessary information below.

If $M$ is a left DG $R$-module and a right DG $T$-module in such a way that $(r m) t=r(m t)$ for all $r \in R, m \in M$, and $t \in T$, then $M$ is called a DG $R$ - $T$-bimodule. We denote this by writing ${ }_{R} M_{T}$. Equivalently, $M$ is a (left) DG $\left(R \otimes_{\mathbb{Z}} T^{\mathrm{op}}\right)$-module with $(r \otimes t) m=(-1)^{|t||m|} r m t$, when $T^{\text {op }}$ denotes the DG ring with $T^{\mathrm{op}}=T$ as DG $\mathbb{Z}$-modules and with product $\cdot$ defined by $t \cdot t^{\prime}=(-1)^{|t|\left|t^{\prime}\right|} t^{\prime} t$. Now consider the following situation: ${ }_{S} L_{R},{ }_{R} M_{T}$, and ${ }_{R} N_{U}$. There are natural structures as follows:

$L \otimes_{R} M$ is a DG $\left(S \otimes_{\mathbb{Z}} T^{\mathrm{op}}\right)$-module by

$(s \otimes t)(l \otimes m)=(-1)^{d}(s l) \otimes(m t)$ with $d=|t|(|l|+|m|) ;$

$\operatorname{Hom}_{R}(M, N)$ is a DG $\left(T \otimes_{\mathbb{Z}} U^{\text {op }}\right)$-module by

$((t \otimes u) \alpha)(m)=(-1)^{e} \alpha(m t) u$ with $e=(|t|+|n|)(|\alpha|+|m|)$.

Here $l \in L, m \in M, s \in S, t \in T, u \in U$, and $\alpha \in \operatorname{Hom}_{R}(M, N)$.

Consider now DG bimodules ${ }_{S} P_{T},{ }_{S} L_{R}$, and ${ }_{R} N_{U}$. There is a canonical map

$$
\omega_{P L N}: \operatorname{Hom}_{S}(P, L) \otimes_{R} N \rightarrow \operatorname{Hom}_{S}\left(P, L \otimes_{R} N\right)
$$

defined by $\omega_{P L N}(\beta \otimes n)(p)=(-1)^{|p||n|} \beta(p) \otimes n$, which is a homomorphism of DG $\left(T \otimes_{\mathbb{Z}} U^{\text {op }}\right)$-modules.

(1.10) Assume that there exist homology isomorphisms (denoted by $\simeq$ since the direction does not matter) as follows:

$P^{\prime} \simeq P$ of DG-projective $D G S$-modules, with $P^{\prime} \quad G$ finite of bounded below type;

$L \simeq L^{\prime}$ of right $D G\left(S \otimes_{\mathbb{Z}} R^{\mathrm{op}}\right)$-modules, with $L^{\prime}$ bounded above;

$N \simeq N^{\prime}$ of $D G$-flat $D G R$-modules, with $N^{\prime}$ bounded above.

In this case $\omega_{P L M}$ is a homology isomorphism. 
Proof. Consider the commutative diagram

$$
\begin{array}{ccc}
\operatorname{Hom}_{S}(P, L) \otimes_{R} N & \stackrel{\omega_{P L N}}{\longrightarrow} & \operatorname{Hom}_{S}\left(P, L \otimes_{R} N\right) \\
\simeq \mid & & \mid \simeq \\
\operatorname{Hom}_{S}(P, L) \otimes_{R} N^{\prime} & \stackrel{\omega_{P L N^{\prime}}}{\longrightarrow} & \operatorname{Hom}_{S}\left(P, L \otimes_{R} N^{\prime}\right) \\
\simeq \mid & & \mid \simeq \\
\operatorname{Hom}_{S}\left(P, L^{\prime}\right) \otimes_{R} N^{\prime} & \stackrel{\omega_{P L^{\prime} N^{\prime}}}{\longrightarrow} & \operatorname{Hom}_{S}\left(P, L^{\prime} \otimes_{R} N^{\prime}\right) \\
\simeq \mid & & \mid \simeq \\
\operatorname{Hom}_{S}\left(P^{\prime}, L^{\prime}\right) \otimes_{R} N^{\prime} & \stackrel{\omega_{P^{\prime} L^{\prime} N^{\prime}}}{\longrightarrow} & \operatorname{Hom}_{S}\left(P^{\prime}, L^{\prime} \otimes_{R} N^{\prime}\right) .
\end{array}
$$

Since the vertical maps are homology isomorphisms by (1.4), and the bottom arrow is an isomorphism by inspection, the top arrow is a homology isomorphism, as claimed.

A G ring or DG ring $R$ is said to be commutative if the following hold for all $r, r^{\prime} \in R: r^{\prime} r=(-1)^{|r|\left|r^{\prime}\right|} \mid r r^{\prime}$ and $r^{2}=0$ if $|r|$ is odd. (Such a ring is sometimes termed strictly commutative.)

When $R$ is commutative every left DG $R$-module can (and will) be considered also as a right DG $R$-module by means of the action: $m r=(-1)^{|m||r|} \mathrm{rm}$ for $m \in M$ and $r \in R$. In particular, the DG $\mathbb{Z}$-modules $\operatorname{Hom}_{R}(M, N)$ and $M \otimes_{R} N$ are then DG $R$-modules for the following actions:

$$
\begin{gathered}
(r \alpha)(m)=(-1)^{|r||\alpha|} \alpha(r m)=(-1)^{|r|(|\alpha|+|m|)} \alpha(m r), \\
r(m \otimes n)=(r m) \otimes n=(-1)^{|r||m|} m \otimes r n,
\end{gathered}
$$

where $r \in R, \alpha \in \operatorname{Hom}_{R}(M, N), m \in M$, and $n \in N$.

From now on (with the exception of (2.12) and (2.13)) all DG rings are assumed to be commutative and concentrated in nonnegative degrees.

\section{BASS SERIES OF FINITE DIMENSIONAL DG ALGEBRAS}

Throughout this section the following assumptions are in force:

- Fdenotes a commutative supplemented $D G$ algebra over a field $\ell$, that is, an augmented $D G$ ring $\varepsilon_{F}: F \rightarrow \ell$ equipped with a homomorphism of $D G$ rings $\eta_{F}: \ell \rightarrow F$ such that $\varepsilon_{F} \eta_{F}=1 /$.

$-\operatorname{dim}_{\ell} F$ is finite.

- $H_{0}(F)$ is a local ring (and then necessarily artinian).

- $f=\max \left\{i \mid H_{i}(F) \neq 0\right\}$.

- $\mathfrak{m}_{H(F)}=\operatorname{ker}\left(H\left(\varepsilon_{F}\right): H(F) \rightarrow \ell\right)$.

For the theory of Golod algebras we refer to [2]. In general, the Golod polynomial of $F$ is defined by

$$
G_{F}(t)=1+t-\sum_{i=0}^{f}\left(\text { length }_{H_{0}(F)} H_{i}(F)\right) t^{i+1} .
$$

Note that, in the setup (2.0), length $H_{0}(F)$ is equal to $\operatorname{dim}_{\ell}$. Recall that the socle of a module over a local ring is the submodule consisting of the elements annihilated by the maximal ideal. 
(2.1) Theorem. Under the assumptions above the following hold.

(a) There is an equality $I_{F}(t)=1$ if and only if $H_{0}(F)$ is a Gorenstein ring and $H_{i}(F)=0$ for $i \neq 0$.

(b) If $\mathfrak{m}_{H(F)} \neq 0$ there is a coefficientwise inequality of formal Laurent series:

$$
I_{F}(t) \ll-t \frac{G_{F}\left(t^{-1}\right)}{G_{F}(t)} .
$$

(c) Equality holds in (b) if and only if $F$ is a Golod algebra and $\mathfrak{m}_{H(F)} \neq 0$.

(d) Both series in (b) have the same order. Their initial terms coincide if and only if $H_{f}(F)=\operatorname{socle}_{H_{0}(F)} H_{f}(F)$ (this is the case, in particular, when $\left.H_{0}(F) \cong \ell\right)$.

(e) depth $F=-f$ and DGtype $F=\operatorname{dim}_{\ell} \operatorname{socle}_{H_{0}(F)} H_{f}(F)$.

Proof. Let $F^{\vee}$ denote the (left) DG $F$-module $\operatorname{Hom}_{l}(F, \ell)$ with operation of $F$ and differential given by, respectively: $(x a)(y)=(-1)^{|x|(|a|+|y|)} a(y x)$ and $(\partial a)(y)=(-1)^{|a|} a(\partial y)$ for $a \in F^{\vee}$ and $x, y \in F$.

We shall make some explicit computations with bar-constructions, cf. [17, (X.10)], so we recall that $\mathbb{B}\left(\ell, F, F^{\vee}\right)=\mathbb{B}(\ell, F, F) \otimes_{F} F^{\vee}$, where $\mathbb{B}(\ell, F, F)$ is defined as follows. Let $\bar{F}$ denote the $\mathrm{G} \ell$-module with $\bar{F}_{i}=(F / \ell)_{i-1}$, and set $(\mathbb{B}(\ell, F, F))^{\#}=\bigsqcup_{p \geq 0}(\bar{F})^{\otimes_{p}} \otimes F^{\#}$ with the action of $F^{\#}$ induced from the right-hand factor. (Tensor products are over $\ell$.) Writing $\left\|y_{1}|\cdots| y_{p}\right\| x$ for a typical element, the differential is defined by

$$
\begin{aligned}
\partial\left(\left\|y_{1}|\cdots| y_{p}\right\| x\right)=-\sum_{i=1}^{p}(-1)^{s^{i-1}}\left\|y_{1}|\cdots| \partial y_{i}|\cdots| y_{p}\right\| x+(-1)^{s^{p}}\left\|y_{1}|\cdots| y_{p}\right\| \partial x \\
\quad+\varepsilon_{F}\left(y_{1}\right)\left\|y_{2}|\cdots| y_{p}\right\| x+\sum_{i=1}^{p-1}(-1)^{s^{i}}\left\|y_{1}|\cdots| y_{i} y_{i+1}|\cdots| y_{p}\right\| x \\
\quad+(-1)^{s^{p}}\left\|y_{1}|\cdots| y_{p-1}\right\| y_{p} x
\end{aligned}
$$

where $s^{i}=i+\sum_{j=1}^{i}\left|y_{j}\right|$.

Note that $H(\mathbb{B}(\ell, F, F)) \cong \ell$ (by the well-known contracting homotopy). That $\mathbb{B}(\ell, F, F)$ is a DG-projective DG $F$-module can easily be verified from its construction, cf. e.g. [5]; thus $H\left(\mathbb{B}\left(\ell, F, F^{\vee}\right)\right)=\operatorname{Tor}^{F}\left(\ell, F^{\vee}\right)$. In particular, it shows that $\operatorname{Tor}_{1}^{F}\left(\ell, F^{\vee}\right)$ is finite dimensional for all $i \in \mathbb{Z}$ and is zero for $i \ll 0$.

By standard isomorphisms we get

$$
\operatorname{Ext}_{F}(\ell, F) \cong \operatorname{Ext}_{F}\left(\ell,\left(F^{\vee}\right)^{\vee}\right) \cong\left(\operatorname{Tor}^{F}\left(\ell, F^{\vee}\right)\right)^{\vee},
$$

and hence

$$
I_{F}(t)=\sum_{i \in \mathbb{Z}}\left(\operatorname{dim}_{\ell} \operatorname{Tor}_{i}^{F}\left(\ell, F^{\vee}\right)\right) t^{i}
$$

Thus $I_{F}(t)$ is a formal Laurent series.

Now, note that $\operatorname{Tor}^{F}\left(\ell, F^{\vee}\right)$ is the abutment of the Eilenberg-Moore spectral sequence obtained from filtering $\mathbb{B}\left(\ell, F, F^{\vee}\right)$ "by the number of bars"

$$
{ }^{2} E_{p q}=\operatorname{Tor}_{p}^{H(F)}\left(\ell, H\left(F^{\vee}\right)\right)_{q} \Rightarrow \operatorname{Tor}_{p+q}^{F}\left(\ell, F^{\vee}\right) .
$$


We denote by $\mathbb{B}$ the ${ }^{1} E$-term of the sequence $(2.3)$ and by $\bar{\partial}$ its differential. Thus, $\mathbb{B}$ is a bigraded vector space with $\mathbb{B}_{p q}=\left(\mathfrak{m}_{H(F)}^{\otimes p} \otimes H\left(F^{\vee}\right)\right)_{q}$. Consider the exact sequence of bigraded spaces

$$
\begin{aligned}
& 0 \rightarrow Z(\mathbb{B}) \rightarrow \mathbb{B} \stackrel{\bar{\partial}}{\rightarrow} \bar{\partial}(\mathbb{B}) \rightarrow 0, \\
& 0 \rightarrow \bar{\partial}(\mathbb{B}) \rightarrow Z(\mathbb{B}) \rightarrow{ }^{2} E \rightarrow 0,
\end{aligned}
$$

where all the maps have bidegree $(0,0)$ except $\bar{\partial}$ which has bidegree $(-1,0)$. We write $|V|(t, u)=\sum_{p, q}\left(\operatorname{dim}_{\ell} V_{p q}\right) t^{p} u^{q}$ for the two-variable Hilbert "series" of a bigraded vector space $V$. Taking into account the equality $\operatorname{dim}_{\ell} H_{i}(F)=$ $\operatorname{dim}_{\ell} H_{-i}\left(F^{\vee}\right)$, we obtain

$$
\begin{aligned}
\left.\right|^{2} E \mid(t, u) & =|\mathbb{B}|(t, u)-(1+t)|\bar{\partial}(\mathbb{B})|(t, u) \\
& =\frac{\sum_{i=0}^{f}\left(\operatorname{dim}_{\ell} H_{i}(F)\right) u^{-i}}{1-t \sum_{i=0}^{f}\left(\operatorname{dim}_{\ell}\left(\mathfrak{m}_{H(F)}\right)_{i}\right) u^{i}}-(1+t)|\bar{\partial}(\mathbb{B})|(t, u) .
\end{aligned}
$$

Next we need some notation and explicit information on the $G H(F)$-module structure of $H\left(F^{\vee}\right)$. The augmentation $\varepsilon_{F}: F \rightarrow \ell$, considered as an element of $F^{\vee}$, is a cycle and gives a nonzero class in $H_{0}\left(F^{\vee}\right)$ : we call it the fundamental class and denote it by $e$. The canonical nondegenerate bilinear map given by the module structure

$$
F \times F^{\vee} \rightarrow F^{\vee} \stackrel{\eta_{F}^{\vee}}{\rightarrow} \ell^{\vee}=\ell
$$

induces in homology an $\ell$-bilinear map of $\mathrm{G} \ell$-modules

$$
H(F) \times H\left(F^{\vee}\right) \rightarrow H\left(F^{\vee}\right) \stackrel{H\left(\eta_{F}^{\vee}\right)}{\longrightarrow} \ell .
$$

This is given by, for $x \in Z_{i}(F)$ and $a \in Z_{j}\left(F^{\vee}\right)$,

$$
([x],[a]) \mapsto\langle[x],[a]\rangle= \begin{cases}x a(1) & \text { if } i+j=0, \\ 0 & \text { if } i+j \neq 0 .\end{cases}
$$

It is also nondegenerate, that is, it is a perfect pairing. Assuming $[x] \neq 0$ and noticing that $\ell[x] \cap\left(\mathfrak{m}_{H(F)}\right)_{0}[x]=0$, it is possible to choose $[a] \in H\left(F^{\vee}\right)$ such that $\langle[x],[a]\rangle=1$ and $\langle[y][x],[a]\rangle=0$ for $[y] \in\left(\mathfrak{m}_{H(F)}\right)_{0}$. Then we have $\langle 1,[x][a]\rangle=1$ and $\langle[y],[x][a]\rangle=0$ for $[y] \in\left(\mathfrak{m}_{H(F)}\right)_{0}$. These equalities characterize $e$, thus $[x][a]=e$ and we obtain:

(2.6) For any nonzero $[x] \in H(F)$ there exists an element $[a] \in H\left(F^{\vee}\right)$ such that $[x][a]=e$.

After these preparations we start proving the different claims of the theorem.

We consider (b) first. Set $T_{p q}=\left(\mathfrak{m}_{H(F)}^{\otimes p}\right)_{q}$ and $T=\bigsqcup_{p, q} T_{p q}$. Remark that viewing $H\left(F^{\vee}\right)$ as a bigraded space with $H\left(F^{\vee}\right)_{0 q}=H\left(F^{\vee}\right)_{q}$ and $H\left(F^{\vee}\right)_{p q}=$ 0 for $p \neq 0$, we have that $\mathbb{B}=T \otimes H\left(F^{\vee}\right)$ as bigraded vector spaces.

Since $\mathfrak{m}_{H(F)} \neq 0$, we can choose $x \in Z\left(\operatorname{Ker} \varepsilon_{F}\right)$ with $[x] \neq 0$ in $\mathfrak{m}_{H(F)}$. Then we use (2.6) to pick an $a \in Z\left(F^{\vee}\right)$ such that $[x][a]=e$. Consider the $\ell$-linear homomorphism of bidegree $(0,0)$

$$
\delta: T \rightarrow \bar{\partial}(\mathbb{B}), \quad \delta(t)=\bar{\partial}(t \otimes[x] \otimes[a]) .
$$


Note that $\delta(t)=t^{\prime} \otimes[a] \pm t \otimes e$ where $t^{\prime} \in T$. Since $[x] e=0$ the elements [a] and $e$ of $H\left(F^{\vee}\right)$ are linearly independent over $\ell$, and thus $\delta$ is injective. Hence

$$
|\bar{\partial}(\mathbb{B})|(t, u) \gg|T|(t, u)=\frac{1}{1-t \sum_{i=0}^{f}\left(\operatorname{dim}_{\ell}\left(\mathfrak{m}_{H(F)}\right){ }_{i}\right) u^{i}} .
$$

Substituting this into $(2.4)$ yields the inequality

$$
\left.\right|^{2} E \mid(t, u) \ll \frac{\sum_{i=0}^{f}\left(\operatorname{dim}_{\ell} H_{i}(F)\right) u^{-i}-(1+t)}{1-t \sum_{i=0}^{f}\left(\operatorname{dim}_{\ell}\left(\mathfrak{m}_{H(F)}\right) u^{i}\right.} .
$$

Combining this with the inequality

$$
I_{F}(t) \ll \sum_{i}\left(\sum_{p+q=i}\left(\operatorname{dim}_{\ell}{ }^{2} E_{p q}\right) t^{i}\right)=\left.\right|^{2} E \mid(t, t),
$$

which follows from (2.2) and the convergence of the spectral sequence (2.3), and with the equality

$$
\sum_{i=0}^{f}\left(\operatorname{dim}_{\ell} H_{i}(F)\right) u^{i}-1=\sum_{i=0}^{f}\left(\operatorname{dim}_{\ell}\left(\mathfrak{m}_{H(F)}\right)_{i}\right) u^{i}
$$

we obtain the required inequality, and the proof of (b) has been completed.

To prove (c) consider the following conditions:

(2.8) $I_{F}(t)=-t G\left(t^{-1}\right) / G(t)$.

(2.9) $\mathfrak{m}_{H(F)} \neq 0$, the homomorphism $\delta$ in (2.7) is surjective, and ${ }^{2} E={ }^{\infty} E$ in the spectral sequence (2.3).

(2.10) $\mathfrak{m}_{H(F)} \neq 0, \mathfrak{m}_{H(F)}^{2}=0$, and ${ }^{2} E={ }^{\infty} E$ in the spectral sequence (2.3).

(2.11) $\mathfrak{m}_{H(F)} \neq 0$ and $F$ is a Golod algebra.

Our claim is that (2.8) and (2.11) are equivalent. In order to establish this, we shall show the equivalence of all four conditions. First, to prove that $(2.8)$ and (2.9) are equivalent, note that (2.8) implies $\mathfrak{m}_{H(F)} \neq 0$, since otherwise it produces the absurd equality $1=I_{F}(t)=-t$. Now the equivalence follows from the argument in the proof of (b).

To prove that (2.9) implies (2.10) assume that there are $u, v \in \mathfrak{m}_{H(F)}$ such that $u v \neq 0$. By (2.6) there is then an $h \in H\left(F^{\vee}\right)$, such that $(u v) h=e$. Since $u e=0$, the elements $v h$ and $e$ of $H\left(F^{\vee}\right)$ are linearly independent over $\ell$. Because of the expression for $\delta$ given in (2.7), this implies that the element $\bar{\partial}(\|v\| h)= \pm v h$ cannot lie in $\operatorname{Im} \delta$. Thus, $\delta$ is not surjective.

To prove that (2.10) implies (2.9) assume that there are $v \in \mathfrak{m}_{H(F)}$ and $h \in H\left(F^{\vee}\right)$, such that $0 \neq v h \in H\left(F^{\vee}\right) / \ell e$. Since in the duality given by (2.5), one has $\left(\mathfrak{m}_{H(F)}\right)^{\perp}=\ell e$, there is a $u \in \mathfrak{m}_{H(F)}$ such that $\langle u, v h\rangle \neq 0$, therefore $u(v h) \neq 0$. But this contradicts the assumption $\mathfrak{m}_{H(F)}^{2}=0$, hence $\mathfrak{m}_{H(F)} H\left(F^{\vee}\right)=\ell e$. It follows that $\bar{\partial}(\mathbb{B})=T \otimes e$. Now the equality $t \otimes e=$ $\pm \delta(t \otimes[x] \otimes[a])$ shows that $\delta$ is surjective.

$(2.10) \Rightarrow(2.11)$. By [2, Theorem (2.3)] we have to show that $H(F)$ has only trivial Massey products. This follows from the argument just after [8, (4.3)] by replacing $H\left(K^{E}\right)$ by $H\left(F^{\vee}\right)$ and shifting the degrees by $-f$.

$(2.11) \Rightarrow(2.8)$. By [2, Theorem 2.3] $F$ is linked by a sequence of homology isomorphisms to a trivial extension $F^{\prime}=\ell \ltimes W$ (that is, $\ell \ltimes W=\ell \oplus W$ as 
vector spaces, $\ell$ is a subring, $W^{2}=0$, and $\left.\partial=0\right)$. Hence $\mathfrak{m}_{H(F)} \cong W$, so in particular $G_{F}(t)=G_{F^{\prime}}(t)$. Furthermore, by (1.8) $I_{F}(t)=I_{F^{\prime}}(t)$. Thus it suffices to prove that there is equality in (2.8) when $F=\ell \ltimes W$. But in this case $(2.10)$ holds trivially, and we use the implication $(2.10) \Rightarrow(2.8)$ which is already available.

To prove (e) note that in the spectral sequence (2.3) we have

$$
{ }^{2} E_{0,-f}=\left(\ell \otimes_{H_{0}(F)} H\left(F^{\vee}\right)\right)_{-f} \cong \ell \otimes_{H_{0}(F)} H_{-f}\left(F^{\vee}\right) \text {. }
$$

Because of the canonical isomorphisms of $H_{0}(F)$-modules

$$
\begin{aligned}
\left(\ell \otimes_{H_{0}(F)} H_{-f}\left(F^{\vee}\right)\right)^{\vee} & =\operatorname{Hom}_{\ell}\left(\ell \otimes_{H_{0}(F)} H_{-f}\left(F^{\vee}\right), \ell\right) \\
& \cong \operatorname{Hom}_{H_{0}(F)}\left(\ell, H_{-f}\left(F^{\vee}\right)^{\vee}\right) \\
& \cong \operatorname{Hom}_{H_{0}(F)}\left(\ell, H_{f}(F)\right) \\
& \cong \operatorname{socle} H_{f}(F),
\end{aligned}
$$

we have $\operatorname{dim}_{\ell}^{2} E_{0,-f}=\operatorname{dim}_{l}$ socle $H_{f}(F)$.

Furthermore $\mathbb{B}_{p q}=0$ when either $p<0$ or $q<-f$. This implies that ${ }^{2} E_{0,-f}={ }^{\infty} E_{0,-f}$, and that ${ }^{\infty} E_{p q}=0$ when either $p+q<-f$ or $p+q=-f$ with $(p, q) \neq(0,-f)$. In other words, the initial term of

$$
I_{F}(t)=\sum_{i \in \mathbb{Z}}\left(\sum_{p+q=i}\left(\operatorname{dim}_{\ell}^{\infty} E_{p q}\right)\right) t^{i}
$$

is $\left(\operatorname{dim}_{\ell}{ }^{2} E_{0,-f}\right) t^{-f}=\left(\operatorname{dim}_{\ell}\right.$ socle $\left.H_{f}(F)\right) t^{-f}$ which was to be proved.

To prove (d) note that, by inspection, the Laurent series $-t G\left(t^{-1}\right) / G(t)$ has initial term (length $\left.{ }_{H_{0}(F)} H_{f}(F)\right) t^{-f}$, hence our claim follows by comparison with the result of (e).

Finally to prove (a) note that the assumption $I_{F}(t)=1$, by $(\mathrm{e})$, implies that $f=0$ and that $\operatorname{dim}_{\ell}$ socle $H_{0}(F)=1$, that is, $H_{i}(F)=0$ for $i \neq 0$ and $H_{0}(F)$ is a Gorenstein ring. Conversely, when the last two conditions are satisfied the spectral sequence $(2.3)$ has ${ }^{2} E_{p q}=0$ for $(p, q) \neq(0,0)$ and ${ }^{2} E_{00} \cong \ell$, hence $I_{F}(t)=1$.

(2.12) Proof of Theorem D. Let $C^{\prime}$ denote a minimal model [19] of the Sullivan algebra of commutative $\mathbb{Q}$-cochains on $G$. The DG rings $C^{*}(G, \mathbb{Q})$ and $C^{\prime}$ are connected by a sequence of homology isomorphisms

$$
\operatorname{Ext}_{C^{*}(G, \mathbb{Q})}\left(\mathbb{Q}, C^{*}(G, \mathbb{Q})\right) \cong \operatorname{Ext}_{C^{\prime}}\left(\mathbb{Q}, C^{\prime}\right),
$$

by (1.5). Furthermore, truncating $C^{\prime}$ if necessary by an ideal of finite codimension and zero cohomology, cf. the proof of (3.4) below, we obtain a homology isomorphism of $C^{\prime}$ onto a finite dimensional connected $\mathbb{Q}$-algebra $C$ with $C^{1}=0$. In particular, by $(1.5)$ again, $\operatorname{Ext}_{C^{\prime}}\left(\mathbb{Q}, C^{\prime}\right)$ and $\operatorname{Ext}_{C}(\mathbb{Q}, C)$ are isomorphic. With due care for the grading convention the arguments of the proof of (2.1) carry over to this cohomological situation and establish the inequality of Theorem $\mathrm{D}$, with equality holding if and only if $H^{*}(G, \mathbb{Q})$ has trivial Massey products. However, this is well known to characterize rational suspensions, $\mathrm{cf}$. [21].

(2.13) Remarks. Let $\ell$ be a field of arbitrary characteristic. For a space $G$ which satisfies the assumption of Theorem $D$, consider now the Betti numbers $b_{i}=\operatorname{dim}_{\ell} H^{i}(G, \ell)$, the cochain algebra $C^{*}(G, \ell)$, and the Bass series 
$I_{G}(t)=\sum_{i \in \mathbb{Z}}\left(\operatorname{dim}_{\ell} \operatorname{Ext}_{C^{*}(G, \ell)}^{-i}\left(\ell, C^{*}(G, \ell)\right)\right) t^{i}$, where the $C^{*}(G, \ell)$-modules under inspection are right modules.

(a) The inequalities of Theorem $\mathrm{D}$ hold in this broader setup.

To see this, replace in the proof of this theorem the Sullivan model of $G$ by a free model, also denoted $C^{\prime}$, of $C^{*}(G, \ell)$ : cf. e.g. [9]. As above one then links $C^{*}(G, \ell)$ by a sequence of homology isomorphisms to a finite dimensional supplemented DG algebra $C$, with $C^{0}=\ell$ and $C^{1}=0$. Such a $C$ will (in general) be noncommutative, but the commutativity of $F$ was never used in the proof of Theorem (2.1.b).

(b) The right-hand side inequality of Theorem $\mathrm{D}$ is an equality if and only if the Massey products of all orders (including the cup products) of elements of $H^{+}(G, \ell)$ are equal to zero.

To prove this one cannot use the argument of Theorem (2.1.c) since the commutativity of $F$ was essential in its last portion. However, the claim can be established by adapting the techniques used in $[8, \S \S 3$ and 4].

(c) If $G$ is a suspension, then there is an identity

$$
I_{G}(t)=\left(\sum_{i=2}^{f} b_{i} t^{-i}-t^{-1}\right) /\left(1-\sum_{i=2}^{f} b_{i} t^{i-1}\right) .
$$

Namely, $H^{*}(G, \ell)$ has trivial Massey products, cf. Kraines [15].

\section{DG FIBERS OF HOMOMORPHISMS OF COMMUTATIVE DG RINGS}

For a $G \mathbb{Z}$-module $B$ we denote by $\Lambda B$ the free commutative $G$ ring on $B$, that is, the (possibly infinite) tensor product of the symmetric algebras on $B_{2 i}$ and exterior algebras on $B_{2 i+1}$ for $i \in \mathbb{Z}$. Every homomorphism of DG rings $\phi: R \rightarrow S$ can be factored in the form

$$
R \underset{\imath}{\hookrightarrow} \underset{\pi_{Y}}{\stackrel{\simeq}{\rightarrow}} S
$$

where $Y^{\#}=R^{\#} \otimes_{\mathbb{Z}} \Lambda B, B$ is concentrated in nonnegative degrees, $B_{n}$ is $\mathbb{Z}$-free for each $n$, and $l$ is the canonical inclusion; this follows from a modification, cf. [3], of the procedure of adjoining variables to kill cycles, cf. [20]. We call such a factorization a free $R$-algebra resolution of $S$; by abuse of language, we give $Y$ the same name. For proofs of the next two properties of the construction we refer to [3] and [20], respectively.

(3.1) $\pi_{Y}$ is a DG-projective resolution of the $D G R$-module $S$.

(3.2) If $\varepsilon_{R}: R \rightarrow \ell$ is surjective, $H_{0}(R)$ is a noetherian ring, and $H_{i}(R)$ is a noetherian $H_{0}(R)$-module for each $i \in \mathbb{Z}$, then $B_{i}$ can be taken of finite rank for each $i \in \mathbb{Z}$. In particular, the $D G R$-module $k$ has a $D G$-projective resolution which is $G$ finite of bounded below type.

Now consider the commutative diagram

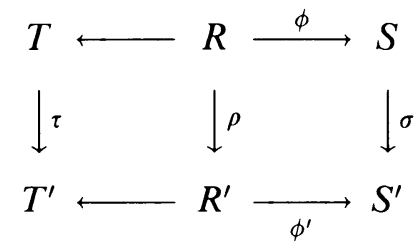


of homomorphisms of commutative DG rings. Embed (say) the right-hand square into the commutative diagram

$$
\begin{array}{ccccc}
R & \hookrightarrow & Y & \underset{\pi_{Y}}{\simeq} & S \\
\downarrow \rho & & \downarrow x & & \downarrow \sigma \\
R^{\prime} & \hookrightarrow & Y^{\prime} & \underset{\pi_{Y^{\prime}}}{\leftrightarrows} & S^{\prime}
\end{array}
$$

This is easily done, extending $\rho$ by induction on the degrees of the elements in a basis of $B$. Thus one obtains a homomorphism of commutative DG rings

$$
\tau \otimes_{\rho} \chi: T \otimes_{R} Y \rightarrow T^{\prime} \otimes_{R^{\prime}} Y^{\prime} .
$$

Since $Y$ (respectively: $Y^{\prime}$ ) can be shown to be a DG-projective resolution of the DG $R$-module $S$ (respectively: of the DG $R^{\prime}$-module $S^{\prime}$ ) this homomorphism represents a map

$$
\tau \underline{\underline{\otimes}}_{\rho} \sigma: T \underline{\underline{\otimes}}_{R} S \rightarrow T^{\prime} \underline{\underline{\otimes}}_{R^{\prime}} S^{\prime}
$$

If one takes algebra resolutions $X$ and $X^{\prime}$ in place of $Y$ and $Y^{\prime}$, respectively, there results a diagram of DG ring homomorphisms

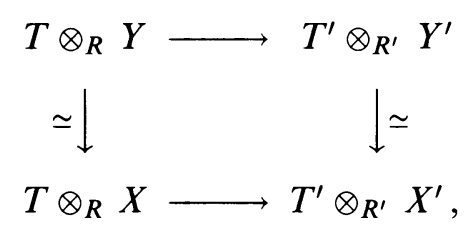

where the vertical maps are homotopy isomorphisms defined uniquely up to homotopy, and which is itself commutative up to homotopy. Due to (1.6), this shows in particular that $\operatorname{Tor}^{-}(-,-)$is a functor from diagrams of the form (3.3) to $\mathrm{G}$ rings.

Definition. If $(R \rightarrow \ell) \rightarrow(S \rightarrow \ell)$ is a homomorphism of augmented DG rings, then any representative of $\ell \underline{\underline{\otimes}}_{R} S$, constructed as above from a free $R$ algebra resolution $Y$ of $S$, will be called a $D G$ fiber of $\phi$, and denoted $F(\phi)$.

The DG fiber $F=F(\phi)$ of $\phi$ will always be considered as an augmented DG ring by means of the augmentation

$$
\varepsilon_{F}=k \underline{\underline{\otimes}}_{\varepsilon_{R}} \varepsilon_{s}: k \underline{\underline{\otimes}}_{R} S \rightarrow \ell \underline{\underline{\otimes}} l=k \otimes_{\ell} \ell=\ell .
$$

The $\mathrm{G} \ell$-module $\operatorname{Ext}_{F}(\ell, F)$ is defined uniquely up to a unique isomorphism: this follows from the preceding discussion and (1.6).

Remark. The fiber of a local homomorphism $\phi$ of local rings (or, more generally, of augmented $\Gamma$-DG rings, that is, DG rings with divided powers) was introduced in [1] as a $\Gamma$-DG ring and called the homotopy fiber. The construction described above gives a DG fiber in the category of augmented DG rings (no divided powers). However, both fibers are homology isomorphic, hence by (1.8) the $\ell$-vector spaces $\operatorname{Ext}_{F}(\ell, F)$ are canonically isomorphic for any choice of the fiber $F$. In particular, the Bass "series" $I_{F}(t)$ does not depend on the choice of the fiber $F$. We denote it by $I_{\phi}(t)$ and call it the Bass series of 
$\phi$. Furthermore, we set depth $\phi=\operatorname{depth} F(\phi)$ and DGtype $\phi=$ DGtype $F(\phi)$, cf. (1.8).

One of the main advantages of the DG version of homological algebra, as compared with the classical one, is the possibility of changing a ring argument by a homology isomorphic one, which may be more suitable for the computations at hand. The next two propositions provide efficient ways of using finiteness assumptions on the DG fiber. by

For a DG $A$-module $N$ the flat dimension of $N, \mathrm{fd}_{A} N$, over $A$ is defined

$$
\mathrm{fd}_{A} N=\inf _{F} \sup \left\{n \mid F_{n} \neq 0\right\}
$$

where the infimum is taken over all DG-flat DG $R$-modules $F$ such that there exists a sequence of homology isomorphisms between $N$ and $F$.

(3.4) Proposition. Let $\phi: R \rightarrow S$ be a homomorphism of augmented $D G$ rings, $R \rightarrow \ell$ and $S \rightarrow \ell$. Assume furthermore that

(a) $R$ is concentrated in degree zero,

(b) $S$ is a $D G R$-module of finite flat dimension.

Then for any free R-algebra resolution: $R \rightarrow Y \underset{\pi_{Y}}{\stackrel{\cong}{S}} S$ there exists a $D G$ ideal $J$ in $Y$ such that the surjection $Y \rightarrow Y^{\prime}=Y / J$ is a homology isomorphism, all $Y_{i}^{\prime}$ are flat $R$-modules, and $Y_{i}^{\prime}=0$ for $i<0$ and for $i>\mathrm{fd}_{R} S$.

Furthermore, there is a canonical isomorphism:

$$
\operatorname{Ext}_{F(\phi)}(\ell, F(\phi)) \cong \operatorname{Ext}_{\ell \otimes_{R} Y^{\prime}}\left(\ell, \ell \otimes_{R} Y^{\prime}\right) .
$$

Remark. Recall that all DG rings are assumed to be commutative and concentrated in nonnegative degrees.

Proof. Let $f=\mathrm{fd}_{R} S$. It follows from the definition of flat dimension that $H_{i}(Y) \cong H_{i}(S)=0$ for $i>f$.

First we prove that $Y_{f} / B_{f}(Y)$ is a flat $R$-module. Note that

$$
\cdots \rightarrow Y_{f+2} \rightarrow Y_{f+1} \rightarrow Y_{f} \rightarrow 0 \rightarrow \cdots
$$

is a flat resolution of $Y_{f} / B_{f}(Y)$, and hence

$$
\operatorname{Tor}_{1}^{R}\left(M, Y_{f} / B_{f}(Y)\right)=H_{f+1}\left(M \otimes_{R} Y\right)=\operatorname{Tor}_{f+1}^{R}(M, S)=0,
$$

for all $R$-modules $M$.

Consider the subcomplex $J$ of $Y$

$$
J=\cdots \rightarrow Y_{f+2} \rightarrow Y_{f+1} \rightarrow B_{f}(Y) \rightarrow 0 \rightarrow \cdots .
$$

This is a DG ideal in $Y$, and $H(J)=0$. Thus the canonical projection $\kappa: Y \rightarrow$ $Y / J=Y^{\prime \prime}$ is a homology isomorphism of DG rings, and $Y^{\prime \prime}$ is $R$-flat (since $Y_{f+1} / B_{f+1}(Y)$ is flat).

The homology isomorphism $\kappa$ induces a homology isomorphism $k \otimes \kappa$ : $k \otimes_{R} Y \rightarrow k \otimes_{R} Y^{\prime \prime}$, by (1.4). Now, note that $F(\phi)$ is equal to $k \otimes_{R} Y$ and apply (1.5) to obtain the isomorphism in Ext.

(3.5) [3, (10.6)] Let $\varepsilon_{G}: G \rightarrow \ell$ be an augmented $D G$ ring such that $H_{0}(G)$ is an artinian local ring which contains a field $k$ as a subring. If $H(G)$ has finite length over $H_{0}(G)$ then $G$ can be linked by a sequence of homology isomorphisms to a supplemented finite dimensional $D G \ell$-algebra $\varepsilon_{F}: F \rightarrow \ell$. 


\section{A BASE CHANGE FORMULA FOR COHOMOLOGY}

In this section we prove the main result of the paper.

(4.1) Theorem. Let $R \rightarrow \ell$ and $S \rightarrow \ell$ be augmented commutative DG rings with surjective augmentations, let $\phi: R \rightarrow S$ be a homomorphism of augmented $D G$ rings, and let $M$ be a DG R-module. Assume furthermore that:

(a) $R$ is a noetherian ring concentrated in degree zero;

(b) $H_{0}(S)$ is a noetherian ring and $H_{i}(S)$ is a finitely generated $H_{0}(S)$ module for each $i \in \mathbb{Z}$

(c) $S$ is a DG R-module of finite flat dimension;

(d) $H(M)$ is bounded above.

When $F(\phi)$ denotes the $D G$ fiber of $\phi$, there is an isomorphism of $G \quad \ell$ modules $\operatorname{Ext}_{S}\left(\ell, M \underline{\underline{\otimes}}_{R} S\right) \cong \operatorname{Ext}_{R}(k, M) \otimes_{k} \operatorname{Ext}_{F(\phi)}(\ell, F(\phi))$.

Remark. The proof takes the rest of this section. For an overview of the argument the reader could consult the note [6].

Proof. First of all we introduce resolutions of the different modules and algebras involved. Most of this information is summarized in the following two commutative diagrams of homomorphisms of DG rings (diagrams (I) and (II)):
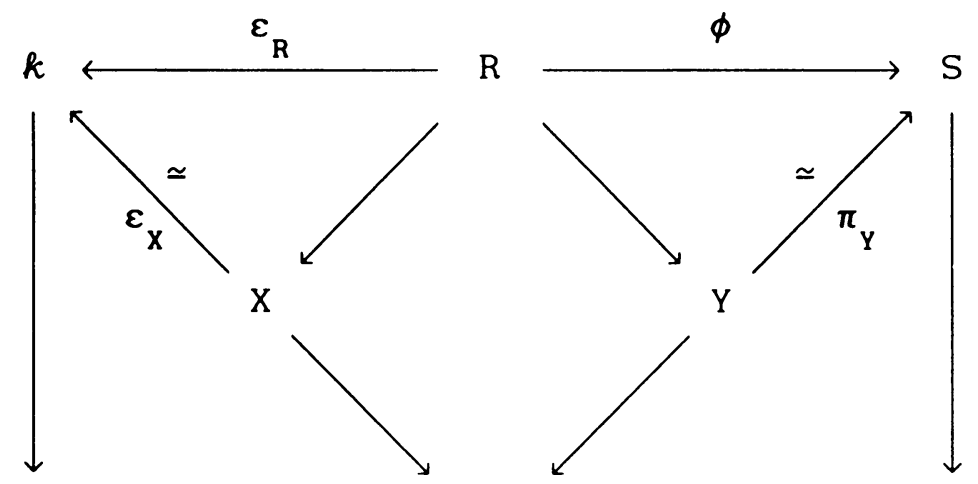

(I)

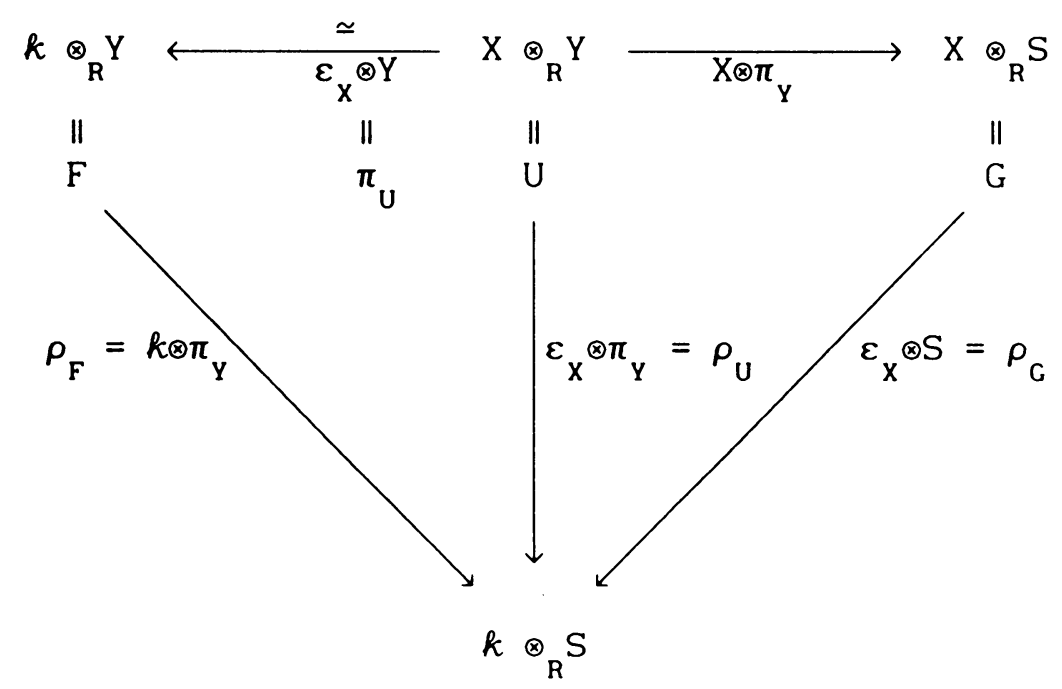


(II)

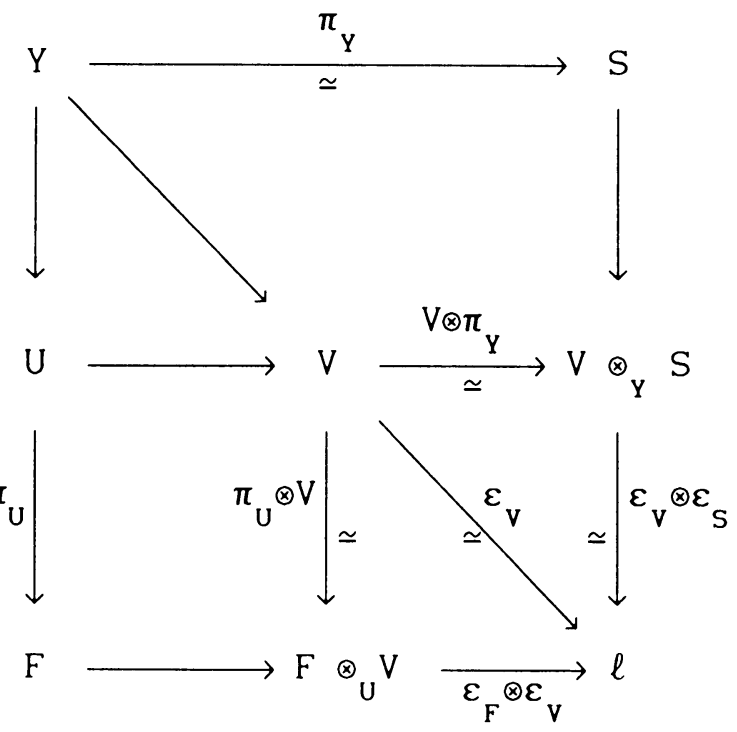

These maps are defined as follows:

$\varepsilon_{X}: X \rightarrow k$ is a free $R$-algebra resolution of $k$ over $R$.

$\pi_{Y}: Y \rightarrow S$ is a free $R$-algebra resolution of $S$.

The equalities define $F, U, G, \rho_{F}, \rho_{U}, \rho_{G}$, and $\pi_{U}$. Note that $F$ is the DG fiber $F(\phi)$ from the preceding section.

$\varepsilon_{V}: V \rightarrow \ell$ is a free $U$-algebra resolution of $\ell$.

Since $\operatorname{Hom}_{Y}\left(X \otimes_{R} Y,-\right) \cong \operatorname{Hom}_{R}(X,-)$ we have that $U$ is a DG-projective DG $Y$-module. By (1.7) this implies:

(4.2) $V$ is a DG-projective DG $Y$-module.

There are several maps that are marked as homology isomorphisms in the diagrams, but not constructed as such. We now give the reasons.

$V \otimes \pi_{Y}$ is a homology isomorphism because $\pi_{Y}$ is a homology isomorphism and $V$ is a DG-projective DG $Y$-module by (4.2).

By the commutativity of the triangle we obtain

(4.3) $\varepsilon_{V} \otimes \varepsilon_{S}$ is a homology isomorphism.

$\pi_{U}=\varepsilon_{X} \otimes Y$ is a homology isomorphism because $\varepsilon_{X}$ is a homology isomorphism and $Y$ is a free DG $R$-module.

$\pi_{U} \otimes V$ is a homology isomorphism because $\pi_{U}$ is a homology isomorphism and $V$ is a DG-projective DG $U$-module.

By the commutativity of the triangle we obtain

(4.4) $\varepsilon_{F} \otimes \varepsilon_{V}$ is a homology isomorphism.

Having explained the diagrams we proceed to record the precise statements needed in the proof.

The canonical isomorphism $\operatorname{Hom}_{S}\left(V \otimes_{Y} S,-\right) \cong \operatorname{Hom}_{Y}(V,-)$ together with (4.2) show that $V \otimes_{Y} S$ is a DG-projective DG $S$-module. Thus (4.3) shows that the following holds.

(4.5) $V \otimes_{Y} S$ is a $D G$-projective resolution of $l$ over $S$.

Next we note that $k \otimes_{X} V$ is, in a natural way, a DG module over $k \otimes_{X} U \cong$ $k \otimes_{R} Y=F$, and that 
(4.6) The canonical isomorphisms

$$
\not \otimes_{X} V \cong h \otimes_{X} U \otimes_{U} V \cong h \otimes_{R} Y \otimes_{U} V \cong F \otimes_{U} V
$$

are $F$-linear.

Now, since $\operatorname{Hom}_{F}\left(F \otimes_{U} V,-\right) \cong \operatorname{Hom}_{U}(V,-)$ the DG $F$-module $F \otimes_{U} V$ is DG-projective, and hence, due to (4.4), we have

(4.7) $k \otimes_{X} V$ is a DG-projective resolution of $\ell$ over $F$.

We also introduce homology isomorphisms of complexes (DG $R$-modules)

$$
P \stackrel{\simeq}{\longrightarrow} M \stackrel{\cong}{\rightarrow} I
$$

with $P$ DG-projective and $I$ DG-injective and bounded above, cf. (1.1) and (1.9). by

Finally, consider $\operatorname{Hom}_{Y}\left(U, I \otimes_{R} Y\right)$ with the DG $U$-module structure given

$$
(u \alpha)\left(u^{\prime}\right)=(-1)^{d} \alpha\left(u^{\prime} u\right) \quad \text { where } d=|u|\left(|\alpha|+\left|u^{\prime}\right|\right)
$$

for $\alpha \in \operatorname{Hom}_{Y}\left(U, I \otimes_{R} Y\right)$ and $u, u^{\prime} \in U$, and consider $\operatorname{Hom}_{R}\left(X, I \otimes_{R} Y\right)$ with the DG $U$-module structure given by

$$
((x \otimes y) \beta)\left(x^{\prime}\right)=(-1)^{e} y \beta\left(x x^{\prime}\right) \quad \text { where } e=|x|(|\beta|+|y|),
$$

for $\beta \in \operatorname{Hom}_{R}\left(X, I \otimes_{R} Y\right), x, x^{\prime} \in X$, and $y \in Y$.

A straightforward computation shows that

(4.8) The canonical isomorphism $\theta: \operatorname{Hom}_{Y}\left(U, I \otimes_{R} Y\right) \stackrel{\simeq}{\rightarrow} \operatorname{Hom}_{R}\left(X, I \otimes_{R} Y\right)$ is an isomorphism of $D G U$-modules.

Because of condition (a) there is a homology isomorphism $X \cong X^{\prime}$ with $X^{\prime}$ a DG-projective DG $R$-module, which is $G$ finite and of bounded below type. By (3.4) there is a homology isomorphism $Y \cong Y^{\prime}$ with $Y^{\prime}$ a bounded DG-flat DG $R$-module. Thus by $(1.10)$ we conclude that

(4.9) $\operatorname{Hom}_{R}(X, I) \otimes_{R} Y \rightarrow \operatorname{Hom}_{R}\left(X, I \otimes_{R} Y\right)$ is a homology isomorphism of $D G U$-modules.

Now follows a sequence of isomorphisms and homology isomorphisms with explanations in square brackets.

$$
\begin{aligned}
\operatorname{Hom}_{S}\left(V \otimes_{Y} S, P \otimes_{R} S\right) \\
\quad \cong \operatorname{Hom}_{Y}\left(V, P \otimes_{R} S\right) \\
\quad \cong \operatorname{Hom}_{Y}\left(V, P \otimes_{R} Y\right) \\
\quad \cong \operatorname{Hom}_{Y}\left(V, I \otimes_{R} Y\right) \\
\cong \operatorname{Hom}_{U}\left(V, \operatorname{Hom}_{Y}\left(U, I \otimes_{R} Y\right)\right) \\
\cong \operatorname{Hom}_{U}\left(V, \operatorname{Hom}_{R}\left(X, I \otimes_{R} Y\right)\right)
\end{aligned}
$$

[canonical isomorphism] $\left[\begin{array}{c}Y \stackrel{\cong}{\rightrightarrows} S ; P \text { is DG-flat over } R ; \\ V \text { is DG-projective over } Y\end{array}\right]$

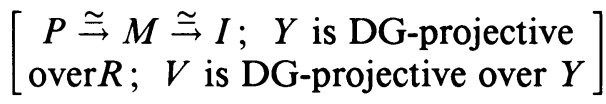
[canonical isomorphism] 


$$
\begin{aligned}
& \cong \operatorname{Hom}_{U}\left(V, \operatorname{Hom}_{R}(X, I) \otimes_{R} Y\right) \\
& \cong \operatorname{Hom}_{U}\left(V, \operatorname{Hom}_{R}(\not k, I) \otimes_{R} Y\right) \\
& \cong \operatorname{Hom}_{F}\left(F \otimes_{U} V, \operatorname{Hom}_{R}(\not k, I) \otimes_{R} Y\right) \\
& \cong \operatorname{Hom}_{F}\left(k \otimes_{X} V, \operatorname{Hom}_{R}(\not k, I) \otimes_{R} Y\right) \\
& \cong \operatorname{Hom}_{F}\left(k \otimes_{X} V, \operatorname{Hom}_{R}(\not k, I) \otimes_{k} F\right)
\end{aligned}
$$

(4.9) since $V$ is DG-projective over $U$ $X \cong k$;

$I$ is DG-injective over $R$; $Y$ is DG-projective over $R$; $V$ is DG-projective over $U$ [canonical isomorphism] $\left[\begin{array}{c}\text { canonical isomorphism } \\ \text { using } F=k \otimes_{R} Y\end{array}\right]$

Now we note that $H_{0}(F)=k \otimes_{R} H_{0}(S)$ is a noetherian ring. Let $X^{\prime} \rightarrow k$ be a resolution by finitely generated projective $R$-modules. Then there is a standard first quadrant spectral sequence of the double complex $X^{\prime} \otimes_{R} S$,

$$
{ }^{2} E_{p q}=H_{p}\left(X^{\prime} \otimes_{R} H_{q}(S)\right) \Rightarrow H_{p+q}\left(X^{\prime} \otimes_{R} S\right) \cong H_{p+q}(F) .
$$

Since ${ }^{2} E_{p q}$ is finitely generated over $H_{0}(S)$ by condition (b), this shows that $H_{i}(F)$ is finitely generated over $H_{0}(F)$ for each $i \in \mathbb{Z}$.

Because of (3.2), the preceding implies that $\ell$ has over $F$ a $D G$-projective resolution $V^{\prime}$, which is $G$ finite and of bounded below type. Since by (4.7) $k \otimes_{X} V$ also is a DG-projective resolution of $\ell$ over $F$, there is a homology isomorphism $V^{\prime} \stackrel{\cong}{\rightrightarrows} \not \otimes_{X} V$ of DG $F$-modules, cf. (1.2). Furthermore, since $H(F)=H\left(k \otimes_{R} Y\right)=\operatorname{Tor}^{R}(k, S)$ is bounded above by condition (c), there is by (3.4) a homology isomorphism $F \cong F^{\prime}$, with $F^{\prime}$ a bounded DG $k$-algebra. Thus we can apply (1.10) to obtain the homology isomorphism

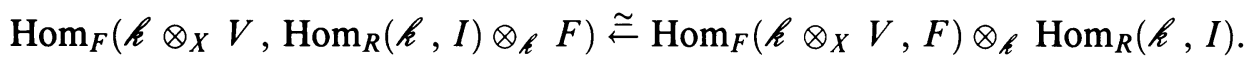

To summarize this

(4.10) There is an isomorphism of the homology of the DG modules

$$
\operatorname{Hom}_{S}\left(V \otimes_{Y} S, P \otimes_{R} S\right) \text { and } \operatorname{Hom}_{F}\left(\ell \otimes_{X} V, F\right) \otimes_{\ell} \operatorname{Hom}_{R}(\not{k}, I) \text {. }
$$

The next sequence of equalities and isomorphisms proves the theorem.

$$
\begin{aligned}
& \operatorname{Ext}_{S}\left(\ell, M \stackrel{\otimes_{R}}{=} S\right)=\operatorname{Ext}_{S}\left(\ell, P \otimes_{R} S\right) \\
& =H\left(\operatorname{Hom}_{S}\left(V \otimes_{Y} S, P \otimes_{R} S\right)\right) \\
& \cong H\left(\operatorname{Hom}_{F}\left(k \otimes_{X} V, F\right) \otimes_{k} \operatorname{Hom}_{R}(k, I)\right) \\
& \cong H\left(\operatorname{Hom}_{F}\left(\not k \otimes_{X} V, F\right)\right) \otimes_{\not} H\left(\operatorname{Hom}_{R}(\not k, I)\right) \\
& \left.=\operatorname{Ext}_{F}(\ell, F) \otimes_{\ell} \operatorname{Ext}_{R}(\not), M\right) \\
& \text { [(4.7) and (1.10)]. }
\end{aligned}
$$

\section{LOCAL HOMOMORPHISMS OF FINITE FLAT DIMENSION}

This section contains the applications to local algebra of the homological computations of the preceding sections. Theorem $\mathrm{A}$ of the Introduction is the special case $M=R$ of (5.1) below, while Theorem B and Theorem $\mathrm{C}$ are obtained from (5.3) and (5.7) in the special case $M=R$. 
Throughout this section the following assumptions will be in force. - $\phi:(R, \mathfrak{m}, k) \rightarrow(S, \mathfrak{n}, \ell)$ is a local homomorphism of finite flat dimension of noetherian local rings, and $\bar{S}=S / \mathrm{m} S$;

- $M$ is a complex of $R$-modules with $H(M)$ bounded above.

Recall that the Bass "series" $I_{R}^{M}(t)$ of $M$ over $R$ is defined by

$$
I_{R}^{M}(t)=\sum_{i \in \mathbb{Z}}\left(\operatorname{dim}_{\mathscr{L}} \operatorname{Ext}_{R}^{i}(\not{k}, M)\right) t^{i} .
$$

For the definitions of the DG fiber $F=F(\phi)$, the Bass "series" $I_{\phi}(t)$, depth $\phi$, and DGtype $\phi$ we refer to (3.2).

(5.1) Theorem. Under the assumptions above $I_{\phi}(t)$ is a nonzero formal Laurent series. Furthermore, if $I_{R}^{M}(t)$ is a formal Laurent series (e.g., if all $H_{i}(M)$ are finitely generated $R$-modules), then $I_{S}^{M} \stackrel{\otimes_{R} S}{=}(t)$ is a formal Laurent series as well, and the following equalities hold:

$$
\frac{I_{S}^{M \otimes}{ }_{R} S}{I_{R}^{M}(t)}=\frac{I_{S}(t)}{I_{R}(t)}=I_{\phi}(t) .
$$

Proof. Consider the isomorphism:

$$
\operatorname{Ext}_{S}\left(\ell, N \underline{\underline{\otimes}}_{R} S\right) \cong \operatorname{Ext}_{R}(\not / N) \otimes_{\ell} \operatorname{Ext}_{F}(\ell, F),
$$

from Theorem (4.1). First we use (1.9) and the isomorphism with $N=R$ to show that $I_{\phi}(t)$ is a nonzero formal Laurent series equal to $I_{S}(t) / I_{R}(t)$, and then we use the isomorphism with $N=M$ to obtain the other equality.

(5.2) Remark. The result applies in particular when $\phi$ is flat and $M$ is a finitely generated $R$-module. In this case the complex $M \underline{\underline{\otimes}}_{R} S$ can be replaced by the module $M \otimes_{R} S$, and the DG fiber $F$ can be replaced by the local ring $\bar{S}$. (Indeed, there are homology isomorphisms: $M \otimes S \rightarrow M \otimes_{R} S$ and $F \rightarrow \bar{S}$, and hence (1.5) applies.) Thus for a flat local homomorphism Theorem (5.1) specializes to the main result of [12].

(5.3) Corollary. The following inequalities hold for all $i \in \mathbb{Z}$

$$
\mu_{R}^{i+\text { depth } R}(M) \leq(\text { DGtype } \phi) \mu_{R}^{i+\text { depth } R}(M) \leq \mu_{S}^{i+\operatorname{depth} S}\left(M \underline{\otimes}_{R} S\right) .
$$

Furthermore, there are equalities

$$
\begin{gathered}
\operatorname{depth}_{S}\left(M \stackrel{\otimes}{=} R_{R} S\right)-\operatorname{depth}_{R} M=\operatorname{depth} S-\operatorname{depth} R=\operatorname{depth} \phi, \\
\frac{\text { DGtype }_{S}\left(M \underline{\otimes}_{R} S\right)}{\text { DGtype }_{R} M}=\frac{\text { DGtype } S}{\text { DGtype } R}=\text { DGtype } \phi .
\end{gathered}
$$

Proof. From the identity $I_{S}(t)=I_{R}(t) I_{\phi}(t)$ it follows that $\operatorname{depth} \phi=\operatorname{depth} S-$ depth $R=d$ and DGtype $F=$ DGtype $S /$ DGtype $R$, that is,

$$
I_{\phi}(t) \gg(\text { DGtype } \phi) t^{d}
$$

and both series have the same initial term. By multiplying both sides by the series with nonnegative coefficients, $I_{R}^{M}(t)$, and applying the theorem once again we see that

$$
I_{S}^{M} \stackrel{\otimes}{R}_{R} S(t)=I_{R}^{M}(t) I_{\phi}(t) \gg(\text { DGtype } F) I_{R}^{M}(t) t^{d}
$$


and the series have the same initial term. This is equivalent to the assertions in the corollary.

(5.4) Remark. Recall that the trivial extension, $R \ltimes N$, of the ring $R$ by an $R$-module $N$, is the abelian group $R \oplus N$ with product $(r, n)\left(r^{\prime}, n^{\prime}\right)=$ $\left(r r^{\prime}, r n^{\prime}+r^{\prime} n\right)$. Let now $N$ be a finitely generated $R$-module of finite projective dimension, and denote by $\phi$ the inclusion $R \hookrightarrow R \ltimes N$. Then $\operatorname{depth} R \ltimes$ $N=\min \left(\operatorname{depth} R, \operatorname{depth}_{R} N\right)$ and $\operatorname{depth} F(\phi)=-\operatorname{pd}_{R} N$ by (3.5) and (2.1.e). Hence it follows from the corollary that $\min \left(\operatorname{depth} R, \operatorname{depth}_{R} N\right)=\operatorname{depth} R-$ $\operatorname{pd}_{R} N$. Thus the Auslander-Buchsbaum formula: $\operatorname{depth}_{R} N+\operatorname{pd}_{R} N=\operatorname{depth} R$ is contained in the identity (5.1) of formal Laurent series.

The next two examples show that the assumption of finite flat dimension in Theorem (5.1) is essential.

(5.5) Example. A local homomorphism $\phi: R \rightarrow S$ with $I_{\phi}(t)=t^{u}$ for some $u$, but $I_{S}(t) \neq I_{R}(t) I_{\phi}(t)$.

Let $x_{1}, \ldots, x_{m}(m>0)$, and $y_{1}, \ldots, y_{n}$ be regular sequences in some local ring $Q$ with maximal ideal $\mathfrak{q}$, such that $x_{i}=\sum_{j=1}^{n} a_{i j} y_{j}$ for $i \in\{1, \ldots, m\}$ and $a_{i j} \in \mathfrak{q}$. Let $\phi: R \rightarrow S$ denote the canonical projection from $R=$ $Q /\left(x_{1}, \ldots, x_{m}\right) \rightarrow S=Q /\left(y_{1}, \ldots, y_{n}\right)$. Let $T_{1}, \ldots, T_{n}$ denote exterior variables of degree 1 , and let $U_{1}, \ldots, U_{m}$ denote divided power variables of degree 2. Finally, let $Y$ denote the DG ring

$$
R\left\langle T_{1}, \ldots, T_{n}, U_{1}, \ldots, U_{m} ; \partial T_{j}=\bar{y}_{j}, \partial U_{i}=\sum_{i=1}^{n} \bar{a}_{i j} T_{j}\right\rangle .
$$

By [20] we have $H_{i}(Y)=0$ for $i \neq 0$ and $H_{0}(Y)=S$, hence $R \rightarrow Y \cong$ $S$ is an algebra resolution of $\phi$. Thus the DG fiber is $F=k \otimes_{R} Y=$ $k\left\langle\bar{T}_{1}, \ldots, \bar{T}_{n}, \bar{U}_{1}, \ldots, \bar{U}_{m}\right\rangle$ with trivial differential. Assume now that $k$ is of characteristic zero; then $F$ is isomorphic to the tensor product $\Lambda \otimes_{k} P$, where $\Lambda$ is the exterior algebra on $\bar{T}_{1}, \ldots, \bar{T}_{n}$ and $P$ is a polynomial algebra on $\bar{U}_{1}, \ldots, \bar{U}_{m}$. We obtain then

$$
I_{\phi}(t)=I_{F}(t)=I_{\Lambda}(t) I_{P}(t)=t^{-n} t^{3 m}=t^{3 m-n} .
$$

On the other hand

$$
I_{S}(t) / I_{R}(t)=I_{Q}(t) t^{-n} / I_{Q}(t) t^{-m}=t^{m-n} \neq I_{\phi}(t) .
$$

(5.6) Example. A local homomorphism $\phi: R \rightarrow S$ with $I_{\phi}(t)=0$.

With $\phi$ as above, assume now that the characteristic of $k$ is $p>0$. Then $F$ is isomorphic to $\Lambda \otimes_{\mathscr{R}} \Gamma$, where $\Gamma$ is the free divided powers algebra on $\bar{U}_{1}, \ldots, \bar{U}_{n}$, which is an infinite tensor product of $\ell$-algebras. Thus $F$ is an infinite tensor product, and this implies $I_{\phi}(t)=0$, cf. [10, proof of (1.7)].

(5.7) Theorem. In the notation of (5.0) assume furthermore, that $I_{R}^{M}(t)$ is a formal Laurent series, and that $\bar{S}$ is artinian.

(a) The equality $I_{S}^{M \otimes_{R}}=(t)=I_{R}^{M}(t)$ holds, if $\phi$ is flat with $\bar{S}$ Gorenstein. Conversely, if $H(M) \neq 0$ and the equality holds, then $\phi$ is flat with $\bar{S}$ Gorenstein. 
(b) Assume that $\phi$ is not flat with $\mathfrak{m} S=\mathfrak{n}$. There is then an inequality

$$
I_{S}^{M \otimes_{R} S}(t) \ll I_{R}^{M}(t) \frac{\sum_{i=0}^{f}\left(\text { length }_{S} \operatorname{Tor}_{i}^{R}(k, S)\right) t^{-i}-t-1}{1+t-\sum_{i=0}^{f}\left(\operatorname{length}_{S} \operatorname{Tor}_{i}^{R}(k, S)\right) t^{i+1}},
$$

where $f=\mathrm{fd}_{R} S$. Equality holds if $\phi$ is Golod. Conversely, if $H(M) \neq 0$ and the equality holds, then $\phi$ is Golod.

(c) There is an inequality:

DGtype $_{S} M \stackrel{\otimes}{=}_{R} S \leq\left(\right.$ DGtype $\left._{R} M\right) \operatorname{length}_{S}\left(\operatorname{Tor}_{f}^{R}(k, S)\right)$,

where $f=\mathrm{fd}_{R} S$. Here, equality holds if and only if $\mathrm{nTor}_{f}^{R}(\not{k}, S)=0$, in particular if $\bar{S}=\ell$.

Proof. First, note that since $H(F) \cong \operatorname{Tor}^{R}(k, S)$, we have $H_{i}(F)=0$ for $i \neq 0$ if and only if $\phi$ is flat; and that $\mathfrak{m}_{H(F)}=0$ if and only if, in addition, $\mathfrak{m} S=\mathfrak{n}$.

Next, note that $I_{R}^{M}(t) \neq 0$ when $H(M) \neq 0$.

Because of (3.5), and the change of rings formula (5.1), assertion (a) follows from (2.1.a), and assertion (b) follows from (2.1.b) and (2.1.c). Assertion (c) is a consequence of (5.3), (2.1.d), and (2.1.e).

(5.8) Remark. Let $Q$ be an arbitrary local ring, and let $\phi: R \rightarrow S$ be a Cohen presentation of the completion $S$ of $Q$, such that the regular local ring $R$ satisfies $\operatorname{dim} R=\operatorname{edim} S$. In this case $\operatorname{Tor}^{R}(\not R, S) \cong H\left(K^{Q}\right)$, where $K^{Q}$ denotes the Koszul complex on a minimal system of generators of the maximal ideal of $Q$. Since $I_{Q}(t)=I_{S}(t)$, Theorem (5.7.b) for $M=R$ coincides with the main result of [8]. When $\phi$ is a surjective Golod homomorphism, the equality in (5.7.b) for $M=R$ was initially established in [16].

(5.9) Proposition. Let $N$ be a nonzero finitely generated $R$-module of finite projective dimension, and let $R \ltimes N$ be the trivial extension. The following identity then holds:

$$
\frac{I_{R \ltimes N}(t)}{I_{R}(t)}=\frac{P_{N}^{R}\left(t^{-1}\right)-t}{1-t P_{N}^{R}(t)}
$$

where $P_{N}^{R}(t)=\sum_{i \geq 0}\left(\operatorname{dim}_{\ell} \operatorname{Tor}_{i}^{R}(\mathcal{E}, N)\right) t^{i}$ is the Poincaré polynomial.

Proof. By [13] $P^{R \ltimes N}(t)=P^{R}(t) /\left(1-t P_{N}^{R}(t)\right)$. Since $1-t P_{N}^{R}(t)=1+t-$ $\sum_{i=0}^{p}\left(\operatorname{dim}_{k} \operatorname{Tor}_{i}^{R}(k, R \ltimes N)\right) t^{i+1}$, where $p$ denotes the projective dimension of the $R$-module $M$, the homomorphism: $R \rightarrow R \ltimes N$ is a standard Golod homomorphism, cf. the Introduction. Thus, the identity is a special case of (5.7.b).

(5.10) Theorem. In addition to the notation of (5.0) set $r=\operatorname{edim} \bar{S}$ and let $x_{1}, \ldots, x_{r}$ be elements of $\mathrm{n}$ which map onto a minimal generating set of $\overline{\mathrm{n}}=$ $\mathrm{n} / \mathrm{m} S$. Let $\phi^{\prime}:\left(R^{\prime}, \mathrm{m}^{\prime}, \ell\right) \rightarrow(S, \mathfrak{n}, \ell)$ be the local homomorphism of $R$ algebra from

$$
R^{\prime}=R\left[X_{1}, \ldots, X_{r}\right]_{\left(\mathfrak{m}+\left(X_{1}, \ldots, X_{r}\right)\right)},
$$

given by $\phi^{\prime}\left(X_{i}\right)=x_{i}$.

(a) The following inequality holds:

$$
\mathrm{fd}_{R^{\prime}} S \leq \mathrm{fd}_{R} S+\operatorname{edim} \bar{S}
$$


If $\bar{S}$ is artinian, then equality holds.

Assume furthermore, that $I_{R}^{M}(t)$ is a formal Laurent series.

(b) The equality $I_{S}^{M{ }^{\otimes}}{ }^{S} S(t)=I_{R}^{M}(t) t^{r}$ holds if $\phi$ is flat with $\bar{S}$ regular. Conversely, if $H(M) \neq 0$ and the equality holds, then $\phi$ is flat with $\bar{S}$ regular.

(c) Assume that $\phi$ is not flat with $\bar{S}$ regular. There is then an inequality:

$$
I_{S}^{M \otimes_{R} S}(t) \ll I_{R}^{M}(t) \frac{-t^{r+1} G_{\phi^{\prime}}\left(t^{-1}\right)}{G_{\phi^{\prime}}(t)},
$$

where $G_{\phi^{\prime}}(t)=1-\sum_{i=1}^{f^{\prime}}\left(\operatorname{dim}_{\ell} \operatorname{Tor}_{i}^{R^{\prime}}(\not{L}, S)\right) t^{i+1}$ for $f^{\prime}=\mathrm{fd}_{R^{\prime}} S$.

The equality holds if $\phi^{\prime}$ is Golod. Conversely, if $H(M) \neq 0$ and the equality holds, then $\phi^{\prime}$ is Golod.

(d) The two series in (c) have the same initial term. In particular, if $H(M) \neq$ 0 then

$$
\operatorname{depth}_{S}(M \underline{\underline{\otimes}} S)-\operatorname{depth} M=\operatorname{edim} \bar{S}-\mathrm{fd}_{R^{\prime}} S \geq-\mathrm{fd}_{R} S .
$$

Proof. We start with the proof of (a). Set $\bar{R}^{\prime}=R^{\prime} / \mathfrak{m} R^{\prime}$. Because of the $R$ flatness of $R^{\prime}$ there are canonical isomorphisms

$$
\operatorname{Tor}_{q}^{R^{\prime}}\left(\bar{R}^{\prime}, S\right) \cong \operatorname{Tor}_{q}^{R}(k, S),
$$

for all $q$, and hence $\operatorname{Tor}_{q}^{R^{\prime}}\left(\bar{R}^{\prime}, S\right)=0$ for $q>\mathrm{fd}_{R} S$. Now the standard change of rings spectral sequence

$$
{ }^{2} E_{p q}=\operatorname{Tor}_{p}^{\bar{R}^{\prime}}\left(\not k, \operatorname{Tor}_{q}^{R^{\prime}}\left(\bar{R}^{\prime}, S\right)\right) \Rightarrow \operatorname{Tor}_{p+q}^{R^{\prime}}(\not{k}, S)
$$

shows that $\operatorname{Tor}_{n}^{R^{\prime}}(k, S)=0$ for $n>\operatorname{fd}_{R} S+\operatorname{edim} \bar{S}$, since $\operatorname{Tor}_{p}^{\bar{R}^{\prime}}(-,-)=0$ for $p>\operatorname{dim} \bar{R}^{\prime}=\operatorname{edim} \bar{S}$, by the regularity of $\bar{R}^{\prime}$. The desired inequality follows, cf. e.g. $[4,(1.7)]$. In the artinian case the equality follows from the usual corner argument in the spectral sequence.

Now we prove (b). Let $F$ denote the DG fiber $F(\phi)$. Because $I^{M \otimes_{R} S}(t)=$ $I_{R}^{M}(t) I_{\phi}(t)$ (by (5.1)), and because $H(M) \neq 0$ implies $I_{R}^{M}(t) \neq 0$, by (1.9), the assertions in (b) follow from the claim

$$
\phi \text { is flat with } \bar{S} \text { regular } \Leftrightarrow I_{\phi}(t)=t^{r} .
$$

For the rest of this proof we set $F^{\prime}=F\left(\phi^{\prime}\right)$. The proof of the claim is divided into three parts.

First we show that

$$
I_{\phi}(t)=t^{r} \Leftrightarrow I_{\phi^{\prime}}(t)=1 .
$$

From (5.1) and (a) we obtain the three identities: $I_{S}(t)=I_{R}(t) I_{\phi}(t), I_{S}(t)=$ $I_{R^{\prime}}(t) I_{\phi^{\prime}}(t)$, and $I_{R^{\prime}}(t)=I_{R}(t) I_{Q}(t)$ where $Q$ is the DG fiber of $R \rightarrow R^{\prime}$. As noted in (5.2) $I_{Q}(t)=I_{\bar{R}^{\prime}}(t)$, which is $t^{r}$ since $\bar{R}^{\prime}$ is regular of dimension $r$. Thus, $I_{\phi}(t)=I_{\phi^{\prime}}(t) t^{r}$, as desired.

Next note that by $(3.5)$ and (2.1.a), since $H_{0}\left(F^{\prime}\right)=\ell$, we have

$$
I_{\phi}(t)=1 \Leftrightarrow \mathfrak{m}_{H\left(F^{\prime}\right)}=0 .
$$


Finally we prove that

$$
\mathfrak{m}_{H\left(F^{\prime}\right)}=0 \Leftrightarrow \phi \text { is flat with } \bar{S} \text { regular. }
$$

Indeed, the isomorphism $H\left(F^{\prime}\right) \cong \operatorname{Tor}^{R^{\prime}}(k, S)$ shows that the vanishing of $\mathfrak{m}_{H\left(F^{\prime}\right)}$ implies that $S$ is $R^{\prime}$-flat, and hence is also $R$-flat. The induced local homomorphisms: $\bar{R}^{\prime} \rightarrow \bar{S}$ is then flat with $\mathfrak{m}^{\prime} S^{\prime}=\overline{\mathfrak{n}}$. Since $\bar{R}^{\prime}$ is regular this shows that $\bar{S}$ is regular. Conversely, if $\phi$ is flat, then the augmentation $F \rightarrow \bar{S}$ is a homology isomorphism, and hence $H\left(F^{\prime}\right)$ is isomorphic to the Koszul homology of $\bar{S}$ with respect to a minimal set of generators of $\overline{\mathfrak{n}}$. This shows that if $\bar{S}$, in addition, is regular then $\mathfrak{m}_{H\left(F^{\prime}\right)}=0$.

Now we turn to the proof of (c). Set $M^{\prime}=M \underline{\underline{\otimes}}_{R} R^{\prime}$. By (a) and (5.7.b) we have the inequality

$$
I_{S}^{M^{\prime}} \stackrel{\otimes_{R^{\prime}} S}{=}(t) \ll I_{R^{\prime}}^{M^{\prime}}(t) \frac{-t G_{\phi^{\prime}}\left(t^{-1}\right)}{G_{\phi^{\prime}}(t)},
$$

with equality if $\phi^{\prime}$ is Golod. By (5.2) we have equalities

$$
I_{R^{\prime}}^{M^{\prime}}(t)=I_{R}^{M}(t) I_{\bar{R}^{\prime}}(t)=I_{R}^{M}(t) t^{r} .
$$

Furthermore, $I_{S}^{M^{\prime}{\stackrel{\otimes}{R^{\prime}}}^{\prime} S}(t)=I_{S} \stackrel{\underline{\otimes}_{R} S}{=} S(t)$ since $M^{\prime} \underline{\otimes}_{R^{\prime}} S$ and $M \underline{\otimes}_{R} S$ are homologically isomorphic. Substitution now yields the desired inequality, which becomes an equality if $\phi^{\prime}$ is Golod.

Finally, to prove (d) note that the initial terms coincide by $(5.1),(3.5)$, and (2.1.d). The equality follows by computing the orders of the series in (c), and the inequality is that of $(\mathrm{a})$.

\section{REFERENCES}

1. L. L. Avramov, Local algebra and rational homotopy, Homotopie Algébrique et Algèbre locale (J.-M. Lemaire and J.-C. Thomas, eds.), Astérisque 113-114 (1984), 15-43.

2. __ Golod homomorphisms, Algebra, Algebraic Topology and their Interactions (J.-E. Roos, ed.), Lecture Notes in Math., vol. 1183, Springer-Verlag, Berlin and New York, 1986, pp. 59-78.

3. __ Locally complete intersection homomorphisms (in preparation).

4. L. L. Avramov and H.-B. Foxby, Locally Gorenstein homomorphisms, Amer. J. Math. 114 (1992).

5. L. L. Avramov, H.-B. Foxby, and S. Halperin, Differential graded homological algebra (in preparation).

6. L. L. Avramov, H.-B. Foxby, and J. Lescot, Séries de Bass des homomorphismes locaux de Tor-dimension finie, C. R. Acad. Paris Sci. Sér. I Math. 309 (1989), 645-659.

7. L. L. Avramov and S. Halperin, Through the looking glass: A dictionary between rational homotopy theory and local algebra, Algebra, Algebraic Topology and their Interactions (J.E. Roos, ed.), Lecture Notes in Math., vol. 1183, Springer-Verlag, Berlin and New York, 1986, pp. 1-27.

8. L. L. Avramov and J. Lescot, Bass numbers and Golod rings, Math. Scand. 51 (1982), 199-211.

9. H. Baues and J.-M. Lemaire, Minimal models in homotopy theory, Math. Ann. 225 (1977), 219-242.

10. Y. Félix, S. Halperin, and J.-C. Thomas, Gorenstein spaces, Adv. Math. 71 (1988), 92-112.

11. H.-B. Foxby, A homological theory of complexes of modules, Københavns Universitets Matematiske Instituts Preprint Series, no. 19, 1981. 
12. H.-B. Foxby and A. Thorup, Minimal injective resolutions under flat base change, Proc. Amer. Math. Soc. 67 (1977), 27-31.

13. T. H. Gulliksen, Massey operations and the Poincaré series of certain local rings, J. Algebra 22 (1972), 223-232.

14. R. Hartshorne, Residues and duality, Lecture Notes in Math., vol. 20, Springer-Verlag, Berlin and New York, 1966.

15. D. Kraines, Massey higher products, Trans. Amer. Math. Soc. 124 (1966), 431-449.

16. J. Lescot, Thèse d'État, Caen, 1985.

17. S. Mac Lane, Homology, Grundlehren Math. Wiss., vol. 114, Springer-Verlag, Berlin, 1967.

18. J. C. Moore, Algèbre homologique et homologie des espaces classifiants, Séminaire H. Cartan, $12^{\mathrm{e}}$ année, exposé 7, (1959-1960), Secrétariat Math., Paris, 1961.

19. D. Sullivan, Infinitesimal computations in topology, Inst. Hautes Études Sci. Publ. Math. 47 (1977), 269-332.

20. J. Tate, Homology of Noetherian rings and local rings, Illinois J. Math. 1 (1957), 14-27.

21. G. Toomer, Lusternik-Schnirelmann category and the Moore spectral sequence, Math. $\mathrm{Z}$. 138 (1974), 123-143.

Institute of Mathematics, ul. "AKad. G. Bončev" Blok 8, BG-1113 Sofia, Bulgaria

Current address: Department of Mathematics, Purdue University, West Lafayette, Indiana 47907

E-mail address: avramov@math.purdue.edu

Københavns Universitets Matematiske Institut, Universitetsparken 5, DK-2100 KøbenhaVN Ø, DenMARK

E-mail address: foxby@math.ku.dk

Département de MathématiQues, Université de Caen, F-14032 Caen cedex, France 